\title{
MULTIFRACTAL SPECTRA FOR RANDOM SELF-SIMILAR MEASURES VIA BRANCHING PROCESSES
}

\author{
J. D. BIGGINS, ${ }^{*}$ University of Sheffield \\ B. M. HAMBLY, ${ }^{* *}$ University of Oxford \\ O. D. JONES, ${ }^{* * *}$ University of Melbourne
}

\begin{abstract}
Start with a compact set $K \subset \mathbb{R}^{d}$. This has a random number of daughter sets, each of which is a (rotated and scaled) copy of $K$ and all of which are inside $K$. The random mechanism for producing daughter sets is used independently on each of the daughter sets to produce the second generation of sets, and so on, repeatedly. The random fractal set $F$ is the limit, as $n$ goes to $\infty$, of the union of the $n$th generation sets. In addition, $K$ has a (suitable, random) mass which is divided randomly between the daughter sets, and this random division of mass is also repeated independently, indefinitely. This division of mass will correspond to a random self-similar measure on $F$. The multifractal spectrum of this measure is studied here. Our main contributions are dealing with the geometry of realisations in $\mathbb{R}^{d}$ and drawing systematically on known results for general branching processes. In this way we generalise considerably the results of Arbeiter and Patzschke (1996) and Patzschke (1997).
\end{abstract}

Keywords: Self-similar measure; Hausdorff dimension; general branching process; multifractal spectrum; local dimension

2010 Mathematics Subject Classification: Primary 28A80; 60J80; 60G18

\section{Introduction}

We give first a fairly informal description of the problem. Start with a compact set $K \subset \mathbb{R}^{d}$. This has a random number of daughter sets, $\left\{K_{i}\right\}$, each of which is a (rotated and scaled) copy of $K$ and all of which are inside $K$, so that $\bigcup_{i} K_{i} \subset K$. The random mechanism for producing daughter sets is used independently on each of the $K_{i}$ to produce the second generation of sets, and so on, repeatedly. The union of the sets forming the $(n+1)$ th generation is, by arrangement, contained in the union of the $n$th generation sets. Hence, it makes sense to take the limit (the intersection) of these as $n$ goes to $\infty$. Then the limit set $F$ can be 'partitioned', using the first generation sets, as $\left\{K_{i} \cap F\right\}$, with each part being a random (scaled) copy of $F$-note that this need not be a true partition because daughter sets may overlap. Now, as an additional feature, $K$ has a (suitable, random) mass which is divided randomly between the $K_{i}$, and this random division of mass is also repeated independently, indefinitely. This mass will derive from a random measure, $\mu$, on $F$, which will be randomly self-similar: $\mu$ can be decomposed into a sum of 'copies' of $\mu$, one defined on each of the sets $K_{i} \cap F$. The law giving the $\left\{K_{i}\right\}$ and the

Received 14 April 2009; revision received 6 December 2010.

* Postal address: School of Mathematics and Statistics, University of Sheffield, Sheffield S3 7RH, UK.

Email address: j.biggins@sheffield.ac.uk

** Postal address: Mathematical Institute, University of Oxford, 24-29 St Giles, Oxford OX1 3LB, UK.

*** Postal address: Department of Mathematics and Statistics, University of Melbourne, Parkville, VIC 3010, Australia. 
division of the mass is called a scaling law. A measure has a local dimension at a point when the logarithm of the measure of a small ball around the point has (in the limit as the ball gets small) a fixed ratio to the logarithm of the volume of the ball. The objective is to study the size, in the sense of the Hausdorff dimension, of the subset of $F$ with a specified local dimension for the self-similar measure associated with a given scaling law. Doing this as the local dimension varies is studying the multifractal spectrum.

We make no attempt to review fully the extensive relevant literature. Initial results on the construction of random self-similar measures on $\mathbb{R}^{d}$ were obtained in [34], and put into the same context as fractal sets in [32]. General results for the existence of a random self-similar measure are due to [15] and [19] or, with the addition of a Markov structure in the construction, [21]. There is a multifractal formalism, developed in the physics literature, which characterises the multifractal spectrum of a set using the Legendre-Fenchel transform of the empirical moment measures. A mathematically precise version can be found in [30]. The application of these ideas to random self-similar measures was the subject of [2], [13], [29], and [31], and is mentioned at the end of [3]. In this paper we establish the usual multifractal spectrum of a random self-similar measure under assumptions that are significantly weaker than those used in [2] by exploiting branching process ideas. In that literature there are already good results (see [3], [4], and [23]) on the multifractal spectrum of random self-similar measures defined on the boundary of trees. There is considerable extra complication in dealing with the geometry of realisations in $\mathbb{R}^{d}$, as we do here.

Despite the description just given, we treat the collection of maps that produce the $K_{i}$ from $K$ as the fundamental object, rather than the sets themselves. The map $S: \mathbb{R}^{d} \rightarrow \mathbb{R}^{d}$ is a contractive similitude when $S y=r O y+x$, where $r<1$ is a contraction factor, $O$ is an orthogonal matrix, and $x$ is a shift. Let $\&$ be the set of contractive similitudes. Now let $A$ be the set of locally finite discrete measures with integer masses on $(0, \infty) \times \S$. A scaling law is a probability measure $\mathrm{P}$ on $A$. For $a \in A$, each unit of mass is interpreted as a point, so that a mass of two gives two points at that place. The points of the process are a set of weights $\left\{p_{i}: i=1,2, \ldots\right\}$ and a corresponding set of similitudes $\left\{S_{i}=\left(r_{i}, O_{i}, x_{i}\right): i=1,2, \ldots\right\}$. Let $N$ be the number of points, so $N \in \mathbb{N} \cup\{\infty\}$. We usually write $\sum_{i}$, or sometimes just $\sum$, for $\sum_{i=1}^{N}$. We assume throughout that $\mathrm{E} \sum p_{i}=1, \mathrm{E} N>1$, and $\mathrm{P}(N=0)=0$.

A scaling law is called compact when there is a compact set $K$ such that $\mathrm{P}\left(\bigcup_{i} S_{i}(K) \subset\right.$ $K)=1$, so all its contractive similitudes map $K$ into $K$. We consider only compact scaling laws. If there is a (nonrandom) finite $c$ such that $\mathrm{P}\left(\sup \left\{\left|x_{i}\right| /\left(1-r_{i}\right): i\right\} \leq c\right)=1$ then the scaling law is compact, for $K$ can be taken to be $\{x:|x| \leq c\}$.

For a given scaling law, a random self-similar measure is one which satisfies

$$
\mu(\cdot)=\sum_{i} p_{i} \mu_{i}\left(S_{i}^{-1}(\cdot)\right) \quad \text { almost surely (a.s.), }
$$

where the $\mu_{i}$ are copies of $\mu$, independent of each other and the scaling law $\left\{\left(p_{i}, S_{i}\right): i=\right.$ $1,2, \ldots\}$, and the supports, $\tilde{F}, \tilde{F}_{i}$, of the measures, $\mu, \mu_{i}$, are random self-similar sets satisfying

$$
\tilde{F}=\bigcup_{i} S_{i}\left(\tilde{F}_{i}\right) \text { a.s. }
$$

We will mainly be interested in the cases where at most one term on the right-hand side of (1.1) contributes at any point. To make this precise, the self-similar measure satisfying (1.1) will be said to partition if there are disjoint sets $V_{i}$ such that $\mu_{i}\left(S_{i}^{-1} V_{i}\right)=\mu_{i}\left(\mathbb{R}^{d}\right)$. Then $\mu\left(V_{i} \cap B\right)=p_{i} \mu_{i}\left(S_{i}^{-1}\left(V_{i} \cap B\right)\right)$. The existence of a self-similar $\mu$ for a scaling law will be 
discussed in the next section, where it will be called $\mu_{\pi}$. It will also emerge later that the measure constructed will partition under suitable conditions. An illustration of the first two steps in the construction of $\tilde{F}$ is given in Figure 1 in Section 4. In this example we can take $V_{i}=\operatorname{int} K_{i}, i=1,2,3$, to obtain a partition.

Let $B(x ; r)$ be the open ball of radius $r$ centred at $x$. We set

$$
\tilde{F}_{\alpha}=\left\{x \in \tilde{F}: \lim _{r \rightarrow 0} \frac{\log \mu(B(x ; r))}{\log r}=\alpha\right\},
$$

which are the points of $\mathbb{R}^{d}$ where the measure of a small ball is, very roughly, its volume to the power $\alpha / d$. The main aim is to estimate the Hausdorff dimension of $\tilde{F}_{\alpha}$. To indicate the results, let

$$
m(q, \phi)=\mathrm{E}\left[\sum_{i} p_{i}^{q} r_{i}^{\phi}\right] \text { and } \beta(q)=\inf \{\phi: m(q, \phi) \leq 1\} .
$$

The Legendre-Fenchel transform of $\beta$ is then

$$
\beta^{*}(\alpha)=\inf _{q}\{\alpha q+\beta(q)\}
$$

this function arises also in the study of the related problem of the growth and spread of a (continuous-time) general branching random walk [7], and that process will play a role here. Let $\mathrm{P}$, which is the scaling law on $A$, also denote the law of self-similar measures induced by that scaling. We will show that, under certain conditions, P-a.s.,

$$
\tilde{F}_{\alpha}=\varnothing \quad \text { if } \beta^{*}(\alpha)<0, \quad \operatorname{dim}_{H}\left(\tilde{F}_{\alpha}\right) \leq \beta^{*}(\alpha) \quad \text { if } \beta^{*}(\alpha) \geq 0,
$$

and that, under other conditions, $\operatorname{dim}_{H}\left(\tilde{F}_{\alpha}\right) \geq \beta^{*}(\alpha)>0$, P-a.s. Obviously, combining results of this kind gives $\operatorname{dim}_{H}\left(\tilde{F}_{\alpha}\right)=\beta^{*}(\alpha)>0$ for suitable $\alpha$. Such results are obtained in [2] and [31]. Both of those treatments assume that $N$ is fixed and there are $p_{\min }$ and $r_{\min }$ with $0<p_{\min }<p_{i}<1$ and $0<r_{\min }<r_{i}<1$. Also, both impose the open set condition (see Definition 2.2): with a suitable $K$, the sets $\left\{K_{i}\right\}$ have nonoverlapping interiors. The strong open set condition adds that there is a positive probability that $F$ is not confined to the boundary of $K$-this is imposed in [2] and relaxed to the open set condition in [31]. Here, generalising these results, $N$ is random, and the uniform bounds on the $\left\{p_{i}\right\}$ and the uniform lower bound on the $\left\{r_{i}\right\}$ are replaced by natural moment conditions. Most results are obtained under the open set condition, though a few do not need it. The strong open set condition is not needed, but part of the treatment is more straightforward when it holds.

An outline of the paper is as follows. In Section 2 we construct our random self-similar measure and give the result concerning its partitioning. In Section 3 the main result is given once enough notation has been established to state it. In Section 4 various preliminary results on branching processes are discussed. In Sections 5 and 8 we derive the main results, giving upper and lower bounds on the multifractal spectrum, that is, on $\operatorname{dim}_{H}\left(\tilde{F}_{\alpha}\right)$. In Section 6 there is a brief discussion about using a single null set for every $\alpha$ in the upper bound. In Section 7 , as part of the lead in to the discussion of lower bounds in Section 8 , the theorem concerning partitioning is proved. We discuss the verification and simplification of some of the conditions in Sections 9 and 10. The final section gives a naturally occurring example drawn from [14] which is easily handled by the results developed here but not by those previously available. 


\section{Random self-similar measures}

We need some standard branching process theory, which we deal with briskly. Let $I_{n}=$ $\bigcup_{k=0}^{n} \mathbb{N}^{k}, I=\bigcup_{k} I_{k}$ and $\bar{I}=I \cup \mathbb{N}^{\mathbb{N}}$. Here $I_{0}$ consists solely of the empty sequence $\varnothing$. A sequence in $\bar{I}$, other than one in $I_{1}$, will be denoted using bold type. Write $\boldsymbol{i} \boldsymbol{j}$ for the concatenation of $\boldsymbol{i} \in I$ and $\boldsymbol{j} \in \bar{I}$. For $\boldsymbol{i} \in \bar{I} \backslash I_{n}$, denote the sequence truncated to length $n$ by $\boldsymbol{i} \mid n$ and the $n$th element of $\boldsymbol{i}$ by $\boldsymbol{i}[n]$. We write $\boldsymbol{j} \leq \boldsymbol{i}$ if $\boldsymbol{i}=\boldsymbol{j} \boldsymbol{k}$ for some $\boldsymbol{k}$, and denote the length of the sequence $i$ by $|\boldsymbol{i}|$. Finally, write $\boldsymbol{i}$ - for the sequence obtained by removing the final entry in $\boldsymbol{i} \in I$, so that $\boldsymbol{i}-=\boldsymbol{i} \mid n$ when $|\boldsymbol{i}|=n+1$.

A tree $T$ is a subset of $I$ such that (i) $\varnothing \in T$ is the root of the tree; (ii) $i \in T$ implies that $\boldsymbol{i} \mid k \in T$ for all $k<|\boldsymbol{i}|$; and (iii) for each $\boldsymbol{i} j \in T, \boldsymbol{i} k \in T$ for $k<j$. Part (iii) does not rule out the possibility that a node produces a countably infinite number of branches. Level $n$ of the tree $T$ will be denoted by $T_{n}=T \cap \mathbb{N}^{n}$. Denote by $\partial T \subset \mathbb{N}^{\mathbb{N}}$ the topological boundary of the tree. We define the cylinder sets of $\partial T$ as follows: for $i \in T_{n}, C_{i}$ is the set of all boundary points stemming from the node $\boldsymbol{i}$, so $C_{\boldsymbol{i}}=\{\boldsymbol{j} \in \partial T: \boldsymbol{j} \mid n=\boldsymbol{i}\}$.

A marked tree has a mark at each of its nodes. Let $\Omega$ be the space of trees with each node marked with an element of $A$. Let $\mathscr{B}_{n}$ be the $\sigma$-algebra generated by the marks at all nodes up to level $n-1$, so that

$$
\mathcal{B}_{n}=\sigma\left(a(\boldsymbol{i}): T \text { a tree, } a \text { a function from } T \text { to } A, i \in T_{k}, k=0,1, \ldots, n-1\right)
$$

and $\mathcal{B}=\sigma\left(\bigcup_{n=1}^{\infty} \mathscr{B}_{n}\right)$. Construct a probability measure, also called $\mathrm{P}$, on $\Omega$ (using the Ionescu Tulcea extension theorem) sequentially in the level of the tree: given the marked tree to level $n$ and $i \in T_{n}$, the mark

$$
a(\boldsymbol{i})=\left(\left(p_{i}(\boldsymbol{i}), S_{i}(\boldsymbol{i})\right): i=1, \ldots\right)=\left(\left(p_{i}(\boldsymbol{i}), r_{i}(\boldsymbol{i}), O_{i}(\boldsymbol{i}), x_{i}(\boldsymbol{i})\right): i=1, \ldots\right)
$$

is used to provide $N(\boldsymbol{i})$, the number of children of $\boldsymbol{i}$, and each of these has an independent mark, drawn from $A$ using law $\mathrm{P}$, attached to it. Note that the marks on the nodes in generation $n-1$ contain the information on the families in generation $n$.

Now it is easy, and common (see [3], [4], [23], and [25]), to define a random self-similar measure on $\partial T$ that relates naturally to the weights in the scaling law. Relevant results are supplied by the theory of the branching random walk (or multiplicative cascades). The connection with that literature is outlined next.

For $i \in T_{n}$, let $p_{i}$ be the product of the values of $p$ associated with $i$ and its antecedents in the tree and let $-z_{i}$ be its logarithm so that, formally,

$$
p_{i}=\prod_{k=0}^{|i|-1} p_{i[k+1]}(i \mid k) \quad \text { and } \quad z_{i}=-\log p_{i} .
$$

Let $Z_{n}$ be the point process on $\mathbb{R}$ with unit mass at the points $\left\{z_{i}: i \in T_{n}\right\}$ (so any $z \in \mathbb{R}$ has an integer mass giving the number $i \in T_{n}$ with $z_{i}=z$ ). Think of individual $i$ having location $z_{i}$. Then $Z=\left\{Z_{n}\right\}_{n=0}^{\infty}$ is a branching random walk (BRW) in the sense of [5], with $Z_{0}$ a unit mass at the origin and offspring displacement given by the point process $Z_{1}$.

Definition 2.1. For a BRW $Z$, let

$$
\tilde{m}(\theta)=\mathrm{E}\left(\int \mathrm{e}^{-\theta t} \mathrm{~d} Z_{1}(t)\right)=\mathrm{E} \sum_{i} p_{i}^{\theta}
$$


and

$$
W^{(n, \theta)}=\tilde{m}(\theta)^{-n} \sum_{i \in T_{n}} \exp \left(-\theta z_{i}\right)=\tilde{m}(\theta)^{-n} \sum_{i \in T_{n}} p_{\boldsymbol{i}}^{\theta} .
$$

Then $W^{(n, \theta)}$ is a positive martingale and, thus, has a P-a.s. limit $W^{(\theta)}$, and, by Fatou's lemma, $\mathrm{E} W^{(\theta)} \leq 1$.

By considering the BRW starting from individual $i \in T_{n}$ we get a martingale limit $W_{i}^{(\theta)}$, with the same distribution as $W^{(\theta)}=W_{\varnothing}^{(\theta)}$ and independent of $W_{j}^{(\theta)}$ for all $\boldsymbol{j} \in T_{n}$ with $\boldsymbol{j} \neq \boldsymbol{i}$. From the branching structure of the BRW we get

$$
W^{(\theta)}=\tilde{m}(\theta)^{-n} \sum_{i \in T_{n}} \exp \left(-\theta z_{i}\right) W_{i}^{(\theta)}=\tilde{m}(\theta)^{-n} \sum_{i \in T_{n}} p_{i}^{\theta} W_{i}^{(\theta)} \quad \text { a.s. }
$$

Note that $\tilde{m}(1)=\mathrm{E} \sum p_{i}=1$ and $\tilde{m}(\theta)=m(\theta, 0)$.

When we have positive finite random variables satisfying $(2.1)$, we can use $W^{(\theta)}$ to define a random measure $\mu^{(\theta)}$ on $\partial T$ by setting

$$
\mu^{(\theta)}\left(C_{\boldsymbol{i}}\right)=\tilde{m}(\theta)^{-n} p_{\boldsymbol{i}}^{\theta} W_{\boldsymbol{i}}^{(\theta)} \quad \text { for } \boldsymbol{i} \in T_{n} .
$$

The existence of such random variables is immediate when $\mathrm{E} W^{(\theta)}=1$ and sharp results for this are known (see [1], [5], [10], and [26]) both for this model and extensions of it. The main cases are described in the next result and translated to the case where $\theta=1$ in the corollary.

Theorem 2.1. (Theorem 7.1 of [10].) Let $Z$ be a BRW, and let $\tilde{m}$ and $W^{(\theta)}$ be given as in Definition 2.1. Then, for all $\theta>0$ such that $\tilde{m}(\theta) \in(0, \infty)$ and $\tilde{m}^{\prime}(\theta)$, which is defined to be $-\mathrm{E}\left(\int t \mathrm{e}^{-\theta t} \mathrm{~d} Z_{1}(t)\right)$, exists, we have $\mathrm{E} W^{(\theta)}=1$ if $\log \tilde{m}(\theta)>\theta \tilde{m}^{\prime}(\theta) / \tilde{m}(\theta)$ and $\mathrm{E} W^{(1, \theta)} \log W^{(1, \theta)}<\infty$.

Corollary 2.1. Suppose that

$$
\mathrm{E} \sum_{i} p_{i}=1, \quad \mathrm{E}\left[-\sum_{i} p_{i} \log p_{i}\right]>0, \quad \text { and } \mathrm{E}\left[\left(\sum_{i} p_{i}\right) \log \left(\sum_{i} p_{i}\right)\right]<\infty .
$$

For $i \in I$, let

$$
W_{i}=\limsup _{n \rightarrow \infty} \frac{1}{p_{\boldsymbol{i}}} \sum_{\left\{\boldsymbol{k}: \boldsymbol{i} \in T_{n}\right\}} p_{\boldsymbol{i} \boldsymbol{k}}
$$

(which is a martingale limit, except on a null set), and $W=W_{\varnothing}$. Then $\mathrm{E} W_{i}=1$. Excluding a suitable P-null set, for $n>|\boldsymbol{i}|$,

$$
p_{i} W_{i}=\sum_{\left\{j: i j \in T_{n}\right\}} p_{i j} W_{i j}
$$

and the measure $\mu\left(=\mu^{(1)}\right)$ in (2.2) is defined on $\partial T$ through $\mu\left(C_{i}\right)=p_{i} W_{i}$. Furthermore, $\mu=\sum p_{i} \mu_{i}$, P-a.s., where $p_{i} \mu_{i}\left(C_{i i}\right)=p_{i i} W_{i i}, \mu_{i}$ is concentrated on $C_{i}$, and, given $\mathcal{B}_{1}$, the $\mu_{i}$ are independent copies of $\mu$.

There are also results on nontrivial solutions to (2.1) under other conditions. When $\log \tilde{m}(\theta)>$ $\theta \tilde{m}^{\prime}(\theta) / \tilde{m}(\theta)>-\infty$ but $\mathrm{E} W^{(1, \theta)} \log W^{(1, \theta)}=\infty$, such results are given in [9]. When $\log \tilde{m}(\theta)=\theta \tilde{m}^{\prime}(\theta) / \tilde{m}(\theta)$ and stronger moment conditions are imposed on $W^{(1, \theta)}$, relevant 
results are given in [22] and [23], and, in some cases, the corresponding self-similar measure on $\partial T$ comes within the study in [3]. We do not consider these other cases and assume that (2.3) always holds from now on. The null set on which (2.4) and $\mu=\sum p_{i} \mu_{i}$ fail is excluded in all that follows.

Fix the compact set $K \subset \mathbb{R}^{d}$ that the scaling law maps into itself. Write $|U|$ for the diameter of $U$ and arrange that $|K|=1$ by the initial choice of units. For $i \in T_{n}$, define $S_{i}$ to be the composition $S_{i[1]}(\varnothing) \circ \cdots \circ S_{i[n]}(\boldsymbol{i} \mid n-1)$ and $K_{i}$, which is geometrically similar to $K$, to be $S_{i}(K)$. Just as for $p_{i}$, let

$$
r_{i}=\prod_{k=0}^{|i|-1} r_{i[k+1]}(i \mid k) \quad \text { and } \quad \sigma_{i}=-\log r_{i} .
$$

Then $\left|K_{i}\right|=r_{i}$. Also, $\sigma_{i}$ can be thought of as the birth time of $\boldsymbol{i}$, and we are in the framework of the general (or Crump-Mode-Jagers) branching process [17, Chapter 6], [28], which for our purposes is just a BRW on $(0, \infty)$.

Lemma 2.1. (Theorem 3 of [8].) If

$$
\text { there exists a } \varphi>0 \text { such that } m(0, \varphi)=\mathrm{E}\left[\sum_{i} r_{i}^{\varphi}\right] \leq 1
$$

then

$$
\inf \left\{\sigma_{i}: i \in T_{n}\right\} \rightarrow \infty \quad\left(\text { and } \sup \left\{r_{i}: i \in T_{n}\right\} \rightarrow 0\right)
$$

P-a.s.

We assume that (2.5) holds. Thus, there is a P-null set, which is excluded in all that follows, such that we have $\sigma_{i \mid n} \rightarrow \infty$ and $r_{i \mid n} \rightarrow 0$ for all $i \in \partial T$, except possibly on this set. A random set $F$ is now defined by

$$
F=\bigcap_{n=1}^{\infty} \bigcup_{i \in T_{n}} K_{i}
$$

Assumption (2.5) ensures that the individual sets here always become small as $n$ gets large.

Following [2], straightforward calculations, using Lemma 2.1 and the fact that the scaling is compact, show that the limit of $S_{i \mid n}(y)$ as $n$ increases exists and is independent of $y \in \mathbb{R}^{d}$ (and is in $K$ ) for each $i \in \partial T$. Thus, $\pi: \partial T \rightarrow \mathbb{R}^{d}$ can be defined by

$$
\pi(\boldsymbol{i})=\lim _{n \rightarrow \infty} S_{\boldsymbol{i} \mid n}(y) .
$$

Furthermore, $\pi$ is continuous. Note that (by taking $y \in K) \pi(\boldsymbol{i})=\bigcap_{n} K_{\boldsymbol{i} \mid n}=\lim _{n \rightarrow \infty} K_{\boldsymbol{i} \mid n}$ and so $\pi$ actually maps $\partial T$ onto $F \subset \mathbb{R}^{d}$. Later it will be natural to use $K_{i}$ as an alternative notation for $\pi(\boldsymbol{i})$. The measure $\mu$ defined in Corollary 2.1 projects into $\mathbb{R}^{d}$ using $\pi$ to give $\mu_{\pi}$. Let $B^{\mathrm{c}}$ be the complement of the set $B$. Then, by definition, $\mu_{\pi}\left(F^{\mathrm{c}}\right)=0$. Recall that $\tilde{F}$ is the support of $\mu_{\pi}$.

Lemma 2.2. We have $F \subset \tilde{F}$. If $\mathrm{P}(N<\infty)=1$ then $F$ is closed and $\tilde{F}=F$.

Proof. By Lemma 2.1, for any $i \in \partial T$ and $\epsilon>0$, there is some $n$ such that $K_{i \mid n} \subset B(\pi(i) ; \epsilon)$ and then $\mu_{\pi}(B(\pi(\boldsymbol{i}) ; \epsilon)) \geq \mu\left(C_{\boldsymbol{i} \mid n}\right)=p_{\boldsymbol{i} \mid n} W_{\boldsymbol{i} \mid n}>0$. Hence, $F \subset \tilde{F}$. Since $\mu_{\pi}\left(F^{\mathrm{c}}\right)=0$, the support of $\mu$ must be contained in the closure of $F$. When $\mathrm{P}(N<\infty)=1$, the collection $T_{n}$ is finite. Then $F$ is closed and so $\tilde{F} \subset F$. 
The measure $\mu_{\pi}$ will be the main object studied here. (The analysis could just as well be applied to $\mu_{\pi}^{(\theta)}$, derived from projecting $\mu^{(\theta)}$ in $(2.2)$, whenever $\mathrm{E} W^{(\theta)}=1$.) From this construction, it is clear that properties of the measure $\mu$ and the set $F$ may be studied via the boundary of the tree $\partial T$.

The first result, which is little more than decoding definitions, is that $\mu_{\pi}$ is indeed selfsimilar, the second is that there is a simple upper bound on the measure of any set. We write $I(A)$ for the indicator function of $A$.

Lemma 2.3. It holds that $\mu_{\pi}$ is self-similar for the scaling law in that, for any $D \subset \mathbb{R}^{d}$,

$$
\mu_{\pi}(D)=\sum_{i} p_{i} \mu_{\pi, i}\left(S_{i}^{-1} D\right),
$$

where, given $\mathcal{B}_{1}$, the $\mu_{\pi, i}$ are independent copies of $\mu_{\pi}$.

Proof. By Corollary 2.1, $\mu$ is self-similar on $\partial T$ with $\mu=\sum p_{i} \mu_{i}$. Now let $\pi_{i}$ be $\pi$ defined using the tree emanating from $i$. Then, for $i \boldsymbol{i} \in C_{i} \subset \partial T, \pi(i \boldsymbol{i})=S_{i} \pi_{i}(\boldsymbol{i})$ and, for any $D \subset \mathbb{R}^{d}$,

$$
\mu_{\pi}(D)=\mu\left(\pi^{-1} D\right)=\sum_{i} p_{i} \mu_{i}\left(\left(\pi^{-1} D\right) \cap C_{i}\right)=\sum_{i} p_{i} \mu_{i}\left(\pi_{i}^{-1} S_{i}^{-1} D\right) .
$$

Now let $\mu_{\pi, i}(\cdot)=\mu_{i}\left(\pi_{i}^{-1} \cdot\right)$, which are, given $\mathscr{B}_{1}$, independent copies of $\mu_{\pi}$, and note that (2.6) then holds.

Lemma 2.4. For $B \subset \mathbb{R}^{d}$ and $n \in \mathbb{N}$,

$$
\mu_{\pi}(B) \leq \sum_{j \in T_{n}} p_{j} W_{j} I\left(B \cap K_{j} \neq \varnothing\right) .
$$

Proof. Note that $\mu\left(C_{\boldsymbol{j}}\right)=p_{\boldsymbol{j}} W_{\boldsymbol{j}}$ and so

$$
\begin{aligned}
\mu_{\pi}(B) & =\mu(\{\boldsymbol{i} \in \partial T: \pi(\boldsymbol{i}) \in B\}) \\
& =\sum_{\boldsymbol{j} \in T_{n}} \mu\left(\{\boldsymbol{i} \in \partial T: \pi(\boldsymbol{i}) \in B\} \cap C_{\boldsymbol{j}}\right), \\
& \leq \sum_{\boldsymbol{j} \in T_{n}} p_{\boldsymbol{j}} W_{\boldsymbol{j}} I\left(\left\{\boldsymbol{j}: \boldsymbol{i} \in C_{\boldsymbol{j}}, \pi(\boldsymbol{i}) \in B\right\}\right) .
\end{aligned}
$$

Furthermore,

$$
\left\{\boldsymbol{j} \in T_{n}: \boldsymbol{i} \in C_{\boldsymbol{j}}, \pi(\boldsymbol{i}) \in B\right\} \subset\left\{\boldsymbol{j} \in T_{n}: B \cap K_{\boldsymbol{j}} \neq \varnothing\right\},
$$

giving (2.7).

At the moment the set $F$ and the measure $\mu$ could be rather simple since, for example, we have not ruled out that all the contractive similarities in the scaling law are the same, in which case $F$ would be a single point. The next condition is a common one for removing this possibility.

Definition 2.2. A scaling law satisfies the open set condition if there is a nonempty bounded open set $O$ with $S_{i}(O) \subset O$ for all $i$ and $S_{i}(O) \cap S_{j}(O)=\varnothing$ when $i \neq j$.

This condition will be needed for most of the results derived. In particular, it will be one of the conditions imposed in showing that the self-similar measure partitions. The next lemma, which is simple to prove, illustrates that it subsumes some of the conditions already mentioned. We write $\operatorname{int}(G)$ or, when there is no ambiguity, $\operatorname{int} G$ for the interior of the set $G \subset \mathbb{R}^{d}$. 
Lemma 2.5. When the open set condition holds, the scaling law is compact and we can (and will) assume that $K$ is the closure of its interior and that the open set condition holds with $O=\operatorname{int} K$. Furthermore, $\sum_{i} r_{i}^{d} \leq 1$, and so (2.5) holds with $\varphi=d$.

The next lemma uses the open set condition to provide a simple basic estimate of $\mu_{\pi}\left(\operatorname{int}\left(K_{i}\right)\right)$. It is worth pointing out that this is only a bound-it is certainly an overestimate when $F$, and, hence, $\mu_{\pi}$ is concentrated on the boundary of $K$. We finish this section by stating the theorem concerning when the measures partition, which will be proved in Section 7.

Lemma 2.6. Under the open set condition, $\mu_{\pi}\left(\operatorname{int}\left(K_{i}\right)\right) \leq p_{i} W_{i}$.

Proof. When $|\boldsymbol{i}|=n$ and $|\boldsymbol{j}|=n$, the open set condition implies that $\operatorname{int}\left(K_{\boldsymbol{i}}\right) \cap K_{\boldsymbol{j}} \neq \varnothing$ if and only if $\boldsymbol{j}=\boldsymbol{i}$. Then, using this observation and (2.7),

$$
\mu_{\pi}\left(\operatorname{int}\left(K_{i}\right)\right) \leq \sum_{j \in T_{n}} p_{j} W_{j} I\left(\operatorname{int}\left(K_{i}\right) \cap K_{j} \neq \varnothing\right)=p_{i} W_{i}
$$

Theorem 2.2. If the open set condition holds then $\mu_{\pi}$ partitions in the sense that, $\mathrm{P}$-a.s., there are disjoint sets $V_{i}$ such that $\mu_{\pi, j}\left(S_{j}^{-1} V_{j}^{\mathrm{c}}\right)=0$ and, for $D \subset \mathbb{R}^{d}$,

$$
\mu_{\pi}(D)=\sum_{i} p_{i} \mu_{\pi, i}\left(S_{i}^{-1}\left(D \cap V_{i}\right)\right)
$$

\section{The main result}

The properties of $m, \beta$, and $\beta^{*}$ have been discussed by several authors (see [2, Section 3], [7, Section 3], and [13, Section 3]), usually under stronger conditions than in force here, and are established with a mixture of calculus and convex analysis drawing on [33]. Convexity of $\log m$ is well known, and follows from Hölder's inequality, as is the concavity of $\beta^{*}$. Two lemmas on convexity are now given: their proofs are deferred to the end of the section. Recall that, by assumption, $m(1,0)=1$; hence, $\beta(1) \leq 0$ and so is finite.

Lemma 3.1. The function $\beta$ is a convex function that is finite somewhere and lower semicontinuous. (It is a proper, closed, convex function in the sense of [33, Section 7].)

These results concerning $\beta$ are given in [7, p. 1011], but with only an indication of the proof. The fact that $\beta$ is lower semicontinuous is important in drawing on general convexity theory-for example, it ensures that the infimum over a bounded convex set is attained [33, Theorem 27.3].

Since $\beta$ is convex and $\beta^{*}$ is concave, we may define $\left(q_{L}, q_{U}\right)$ and $\left(D_{L}, D_{U}\right)$ to be the interior of their respective domains of finiteness. When it makes sense, let

$$
P(q, \phi)=\mathrm{E}\left[-\sum_{i} p_{i}^{q} r_{i}^{\phi} \log p_{i}\right]
$$

and let

$$
R(q, \phi)=\mathrm{E}\left[-\sum_{i} p_{i}^{q} r_{i}^{\phi} \log r_{i}\right]
$$

In the next result, part (vi) is the key to the main proof. It identifies arguments of $m-\mathrm{a}$ $(q, \beta)$-corresponding to a given $\alpha$. The results on $\operatorname{dim}_{H}\left(F_{\alpha}\right)$ are produced via this $(q, \beta)$. 
Lemma 3.2. Assume that $m(1,0)=1, m(0, \varphi)$ is finite for some $\varphi$, and that $P(1,0)$, which is $\mathrm{E}\left[-\sum p_{i} \log p_{i}\right]$, exists and is strictly positive. Then the following statements hold.

(i) If $m(q, \beta)=1$ then $\beta(q)=\beta$. In particular, $\beta(1)=0$.

(ii) $\beta(q)<\infty$ for $0 \leq q \leq 1$, so $(0,1) \subset\left(q_{L}, q_{U}\right)=\operatorname{int}(\{q: \beta(q)<\infty\})$.

(iii) $\beta(q)>0$ for $q<1$.

(iv) When $\beta(q)<\infty, m(q, \beta(q)) \leq 1$.

(v) $\left(D_{L}, D_{U}\right)$ is empty only when there is a constant $\gamma$ such that $p_{i} r_{i}^{-\gamma}=1$ for all $i$-then, $\beta(q)=\gamma(1-q)$ and $\beta^{*}(a)=-\infty$ unless $a=\gamma$ when $\beta^{*}(\gamma)=\gamma$.

(vi) If $\alpha \in\left(D_{L}, D_{U}\right)$ then there is a finite $\tilde{q}(\alpha)$ such that $\beta^{*}(\alpha)=\alpha \tilde{q}(\alpha)+\beta(\tilde{q}(\alpha))$ and $\tilde{q}(\alpha)$ decreases as $\alpha$ increases.

(vii) $\sup \left\{\beta^{*}(\alpha): \alpha\right\}=\beta(0)>0$. Let $\alpha^{\circ}$ be the limit of $q^{-1}(\beta(0)-\beta(q))$ as $q \downarrow 0$ when this exists and is finite, and let $\alpha^{\circ}=\infty$ otherwise. Then this supremum occurs at $\alpha^{\circ}$.

(viii) If $m(q, \phi)=1$ and $\alpha=P(q, \phi) / R(q, \phi)$ makes sense and is finite, then $\beta^{*}(\alpha)=\alpha q+\phi$. In particular, when $m(0, \beta(0))=1, \alpha^{\circ}=P(0, \beta(0)) / R(0, \beta(0))$ when this makes sense and is finite.

We now collect together several conditions already mentioned into Assumption A, given next. In addition to it, we will also need conditions to hold for a particular $(q, \beta)$ and these are contained in the second assumption.

Assumption A. (i) We have a scaling law mapping the compact set $K$ into itself.

(ii) $\mathrm{E} \sum p_{i}=1, \mathrm{E}\left[-\sum p_{i} \log p_{i}\right]>0$, and $\mathrm{E}\left[\sum p_{i} \log \left(\sum p_{i}\right)\right]<\infty$.

(iii) There is a $\varphi>0$ with $\mathrm{E} \sum_{i} r_{i}^{\varphi} \leq 1$.

(iv) $K$ has diameter 1 , and so is contained in a closed ball of radius one-half.

Assumption B. (i) $(q, \beta)$ is such that $m(q, \beta)=\mathrm{E} \sum_{i} p_{i}^{q} r_{i}^{\beta}=1$.

(ii) With $y_{i}=p_{i}^{q} r_{i}^{\beta}, \mathrm{E}\left[-\sum y_{i} \log y_{i}\right]>0$ and $\mathrm{E}\left[\sum y_{i} \log \left(\sum y_{i}\right)\right]<\infty$.

(iii) $P(q, \beta)=-\mathrm{E} \sum_{i} p_{i}^{q} r_{i}^{\beta} \log p_{i}$ exists and is finite.

(iv) $\mathrm{E} \sum_{i} p_{i}^{q} r_{i}^{\beta}\left(\log r_{i}\right)^{2}<\infty$, which implies that $R(q, \beta)<\infty$.

Lemma 3.2(viii) implies that, when Assumption B holds, $\beta(q)=\beta$ and $\beta^{*}(\alpha)=q \alpha+\beta$ with $\alpha=P(q, \beta) / R(q, \beta)$. When Assumption B(i) and $\mathrm{B}$ (ii) hold, Corollary 2.1 applies with ' $y$ ' replacing ' $p$ '. This shows that if we let

$$
Y_{i}=\lim _{n \rightarrow \infty} \frac{\sum_{i k \in T_{n}} y_{i k}}{y_{i}}\left(=\lim _{n \rightarrow \infty} \frac{\sum_{i k \in T_{n}} p_{i k}^{q} r_{i k}^{\beta}}{p_{i}^{q} r_{i}^{\beta}}\right)
$$

and $Y=Y_{\varnothing}$, then $\mathrm{E} Y_{i}=1$ and, for $|\boldsymbol{i}|<n$,

$$
y_{\boldsymbol{i}} Y_{\boldsymbol{i}}=\sum_{\left\{\boldsymbol{j}: \boldsymbol{i} \boldsymbol{j} \in T_{n}\right\}} y_{\boldsymbol{i} \boldsymbol{j}} Y_{\boldsymbol{i} \boldsymbol{j}}=\sum_{\left\{\boldsymbol{j}: \boldsymbol{i} \boldsymbol{j} \in T_{n}\right\}} p_{\boldsymbol{i} \boldsymbol{j}}^{q} r_{\boldsymbol{i} \boldsymbol{j}}^{\beta} Y_{\boldsymbol{i} \boldsymbol{j}}
$$


Define the measure $v$ on $\partial T$ through $v\left(C_{i}\right)=y_{i} Y_{i}$, and let $v_{\pi}$ be the projection of $v$ onto $\mathbb{R}^{d}$, which like $\mu_{\pi}$ has its mass confined to $\pi(\partial T)=F$. This measure will play an important role in the proof, and its associated random variable, $Y$, figures in the conditions. Of course, $\mu$ is an example of a measure of this kind, with $q=1$ and $\beta=0$, and so results obtained for $v$ and $\nu_{\pi}$ will apply to $\mu$ and $\mu_{\pi}$ as particular cases.

For the upper bound on the dimension, we need to have an estimate of the size of the boundary of $K$ in some cases. The estimate used is contained in the next definition.

Definition 3.1. For a compact set $K$ with diameter 1 and $a \leq 1$, let $\hat{h}(a)$ be the maximum number of disjoint open balls with diameter no smaller than $a$ that can intersect the boundary of $K$. Then the set $K$ will be said to have a $\gamma$-boundary when there is a finite $C^{\prime}$ such that $\hat{h}(x) \leq C^{\prime} x^{-\gamma}$ for $x \leq 1$.

If $K$ has a $\gamma$-boundary, it also has a $\gamma^{\prime}$-boundary for all $\gamma^{\prime}>\gamma$, and it is easy to see that when $K$ has a $\gamma$-boundary its boundary has a Hausdorff dimension of no more than $\gamma$. Since $K$ is contained in a closed ball of radius one-half, a crude volume argument shows that $\hat{h}(a)$ cannot be more than $C^{\prime} / a^{d}$ for some constant $C^{\prime}$, but in cases where $K$ has a 'nice' boundary $\hat{h}$ will grow no faster than $C^{\prime} / a^{d-1}$.

We are now ready to state the main theorem. It has no content when $\left(D_{L}, D_{U}\right)$ is empty, which Lemma 3.2(v) shows to be a very special case, but the lemmas leading up to the proof of this theorem do provide information on that case too. By analogy with $\tilde{F}_{\alpha}$, let

$$
F_{\alpha}=\left\{x \in F: \lim _{r \rightarrow 0} \frac{\log \mu_{\pi}(B(x ; r))}{\log r}=\alpha \text { a.s. }\right\}=F \cap \tilde{F}_{\alpha} .
$$

Thus, $F_{\alpha} \subset \tilde{F}_{\alpha}$ and so a lower bound on $\operatorname{dim}_{H}\left(F_{\alpha}\right)$ serves also for $\operatorname{dim}_{H}\left(\tilde{F}_{\alpha}\right)$. However, extra argument (supplied, for example, by Lemma 2.2) is needed to move from an upper bound on $\operatorname{dim}_{H}\left(F_{\alpha}\right)$ to an upper bound on $\operatorname{dim}_{H}\left(\tilde{F}_{\alpha}\right)$.

Theorem 3.1. Suppose that the open set condition and Assumption A hold, and that $K$ has a $\gamma$-boundary. Let $\alpha \in\left(D_{L}, D_{U}\right)$, with $\tilde{q}(\alpha)$ and $\beta(\tilde{q}(\alpha))$ as in Lemma 3.2(vi). Assume that

$$
\mathrm{E} W^{\tilde{q}(\alpha)}<\infty .
$$

If $0<\tilde{q}(\alpha) \leq 1$, assume that

$$
m(\tilde{q}(\alpha), 0)=\mathrm{E} \sum_{i} p_{i}^{\tilde{q}(\alpha)}<\infty .
$$

If $\tilde{q}(\alpha)>1$, assume that

$$
\mathrm{E}\left[\left(\min _{i} r_{i}\right)^{\beta(\tilde{q}(\alpha))-\gamma}\left(\sum_{i} p_{i}\right)^{\tilde{q}(\alpha)}\right]<\infty .
$$

Then, if $\beta^{*}(\alpha) \geq 0$,

$$
\operatorname{dim}_{H}\left(F_{\alpha}\right) \leq \beta^{*}(\alpha) \quad \text { a.s. }
$$

Furthermore, if $\beta^{*}(\alpha)<0$ then (a.s.) $F_{\beta}=\varnothing$ for all $\beta \leq \alpha$ when $\alpha<\alpha^{\circ}$ and $F_{\beta}=\varnothing$ for all $\beta \geq \alpha$ when $\alpha>\alpha^{\circ}$ (with $\alpha^{\circ}$ as in Lemma 3.2(vii)).

Suppose, in addition, that Assumption B holds for $(\tilde{q}(\alpha), \beta(\tilde{q}(\alpha)))$, that

$$
\mathrm{E} Y \log _{+} Y<\infty
$$


and that

$$
\mathrm{E} Y|\log W|<\infty
$$

Then

$$
\operatorname{dim}_{H}\left(F_{\alpha}\right)=\beta^{*}(\alpha) \text { a.s. }
$$

The sets $F_{\alpha}$ can be replaced by $\tilde{F}_{\alpha}$ everywhere in this result when $\tilde{F}=F$.

The conditions here are complex, but mostly natural. Results are noted in Section 9 that allow the moment conditions on $W$ and $Y$ to be traced back to conditions on the scaling law. Various results obtained in Section 10 produce stronger, but easier to check, conditions. One outcome of these will be the following result. It improves on [2, Theorem 4.10(II)] and [31, Theorem 5(II)] in allowing $N, \max _{i} r_{i}^{-1}$, and $\max _{i} p_{i}^{-1}$ to be unbounded, though it cuts down considerably on the generality of Theorem 3.1.

Corollary 3.1. Suppose that the open set condition and Assumption A both hold, and that $p_{i} \in(0,1)$ for all $i$. Suppose that, for some $\epsilon>0$, $\mathrm{E} N^{1+\epsilon}<\infty$ and $\mathrm{P}(N \geq 2)=1$. Suppose also that $\mathrm{E}\left(\min _{i} r_{i}\right)^{-\varrho}, \mathrm{E}\left(\min _{i} p_{i}\right)^{-\varrho}$, and $\mathrm{E}\left(\sum p_{i}\right)^{\varrho}$ are finite for all $\varrho>0$. Take $\alpha \in\left(D_{L}, D_{U}\right)$. Then $F_{\alpha}=\varnothing$ a.s. if $\beta^{*}(\alpha)<0$ and $\operatorname{dim}_{H}\left(F_{\alpha}\right)=\beta^{*}(\alpha)$ a.s. if $\beta^{*}(\alpha) \geq 0$.

We mention also the following result, which is included in [27, Theorem 1.1], but is a side issue for the multifractal spectrum.

Theorem 3.2. When the open set condition holds, $\operatorname{dim}_{H}(F)=\beta(0)=\sup \left\{\beta^{*}(\alpha): \alpha\right\}$.

Proof of Lemma 3.1. Note first that, since $r_{i} \leq 1$ and EN $>1, m(q, \phi)$ is strictly decreasing in $\phi$ when finite and then tends to 0 as $\phi \rightarrow \infty$. If $m\left(q_{1}, \phi_{1}\right) \leq 1$ and $m\left(q_{2}, \phi_{2}\right) \leq 1$, then, using convexity of $\log m$,

$$
m\left(\lambda q_{1}+(1-\lambda) q_{2}, \lambda \phi_{1}+(1-\lambda) \phi_{2}\right) \leq m\left(q_{1}, \phi_{1}\right)^{\lambda} m\left(q_{2}, \phi_{2}\right)^{(1-\lambda)} \leq 1,
$$

which implies that $\beta\left(\lambda q_{1}+(1-\lambda) q_{2}\right) \leq \lambda \phi_{1}+(1-\lambda) \phi_{2}$. Since $\phi_{1} \geq \beta\left(q_{1}\right)$ and $\phi_{2} \geq \beta\left(q_{2}\right)$, but are otherwise arbitrary, this gives convexity of $\beta$. Now let $Q$ be the interior of the domain of finiteness of $\beta$, so that $\beta$ is continuous there, and let $\tilde{q}$ be on the boundary of $Q$. Let $\tilde{\beta}=\liminf \{\beta(q): q \in Q, q \rightarrow \tilde{q}\}$. By convexity, $\beta(\tilde{q}) \geq \tilde{\beta}$ and, for the asserted continuity, it remains to show that $\beta(\tilde{q}) \leq \tilde{\beta}$. Take $q_{n} \in Q$ with $q_{n} \rightarrow \tilde{q}$ and $\beta\left(q_{n}\right) \rightarrow \tilde{\beta}$. For given $\epsilon>0$, let $\Gamma(\epsilon)=\sup \left\{\beta\left(q_{n}\right):\left|q_{n}-\tilde{q}\right|<\epsilon\right\}$. Then, $\Gamma(\epsilon) \rightarrow \tilde{\beta}$ as $\epsilon \downarrow 0$. For $\phi>\Gamma(\epsilon)$, $m\left(q_{n}, \phi\right)<1$. Then $1 \geq m\left(q_{n}, \phi\right) \rightarrow m(\tilde{q}, \phi)$ as $q_{n} \rightarrow \tilde{q}$ using monotone and dominated convergence. Hence, $\beta(\tilde{q}) \leq \phi$. Since $\phi>\Gamma(\epsilon)$ is arbitrary, as is $\epsilon>0$, this implies that $\beta(\tilde{q}) \leq \tilde{\beta}$.

Proof of Lemma 3.2. Since $m(q, \phi)$ is strictly decreasing in $\phi$ when finite, $m(q, \beta)=1$ implies that $\beta(q)=\beta$.

Since $\log m$ is convex, $\log m(\lambda,(1-\lambda) \varphi)$ is finite for all $\lambda \in[0,1]$; hence, $\beta(\lambda)<\infty$ there.

Note that $\log m(q, 0)$ is a convex function of $q$, with value 0 and negative slope $\left(\mathrm{E} \sum_{i} p_{i} \times\right.$ $\left.\log p_{i}\right)$ at $q=1$. Hence, $\log m(q, 0)>0$ for $q<1$ and so $\beta(q)>0$ (but it might be $+\infty$ ).

For the fourth part, use monotone convergence on $1 \geq m(q, \beta(q)+\epsilon)=\mathrm{E} \sum_{i} p_{i}^{q} r_{i}^{\beta(q)+\epsilon}$ as $\epsilon \downarrow 0$.

If $\gamma$ is such that $p_{i} r_{i}^{-\gamma}=1$ for all $i$ then

$$
\sum_{i} p_{i}^{q} r_{i}^{\beta}=\sum_{i} p_{i}^{q} p_{i}^{\beta / \gamma}=\sum_{i} p_{i}^{q+\beta / \gamma}
$$


and so $\beta(q)=\gamma(1-q)$, which gives $\beta^{*}$. Now assume that $\beta^{*}$ is finite at only one point (and $-\infty$ elsewhere). Then $\beta$ is linear. Truncate so that there are no more than $c$ births, and only $i$ with $\left|\log p_{i}\right| \leq c$ and $\left|\log r_{i}\right| \leq c$ are included. Denote truncated entities by a prefixed subscript $c$. Then ${ }_{c} m(q, \phi) \uparrow m(q, \phi)$ and so ${ }_{c} \beta(q) \uparrow \beta(q)$ and ${ }_{c} \beta^{*}(\alpha) \uparrow \beta^{*}(\alpha)$. Hence, ${ }_{c} \beta^{*}$ is also finite at only one point. By arrangement, ${ }_{c} m(q, \phi)$ is finite for all $q$ and $\phi$, and so ${ }_{c} m\left(q,{ }_{c} \beta(q)\right)=1$. We now work with the truncated process. Since $\beta$ is linear,

$$
1=m(q, \beta(q))=\mathrm{E} \sum_{i} p_{i}^{q} r_{i}^{\beta(q)}=\mathrm{E} \sum_{i} p_{i}^{q} r_{i}^{\gamma q-c}=\mathrm{E} \sum_{i}\left(p_{i} r_{i}^{\gamma}\right)^{q} r_{i}^{-c},
$$

which is possible only if $\left(p_{i} r_{i}^{\gamma}\right)$ is always 1 . This is true of any truncated process and, hence, also of the untruncated version.

Since $\beta$ is a closed convex function, this is a consequence of [33, Theorems 12.2, 23.4, and 23.5]. Alternatively, take $\epsilon>0$ with $(\alpha-\epsilon, \alpha+\epsilon) \subset\left(D_{L}, D_{U}\right)$. For all $q$,

$$
\beta^{*}(\alpha-\epsilon) \leq(\alpha-\epsilon) q+\beta(q) \quad \text { and } \quad \beta^{*}(\alpha+\epsilon) \leq(\alpha+\epsilon) q+\beta(q) .
$$

Hence,

$$
\min \left\{\beta^{*}(\alpha-\epsilon), \beta^{*}(\alpha+\epsilon)\right\}+\epsilon|q| \leq \alpha q+\beta(q) .
$$

The left-hand side tends to $\infty$ as $q \rightarrow \pm \infty$. Hence, the infimum of the right-hand side is at a finite $q$, and since $\beta(q)$ is closed and convex, the infimum must be attained (see [33, Theorems 8.1 and 27.3]). This gives $\tilde{q}(\alpha)$. For all $q$,

$$
\alpha q+\beta(q) \geq \alpha \tilde{q}(\alpha)+\beta(\tilde{q}(\alpha))
$$

and so, for $\epsilon>0$,

$$
(\alpha+\epsilon) q+\beta(q)-((\alpha+\epsilon) \tilde{q}(\alpha)+\beta(\tilde{q}(\alpha))) \geq \epsilon(q-\tilde{q}(\alpha)),
$$

which is greater than 0 when $q-\tilde{q}(\alpha)>0$. Hence,

$$
\inf _{q}\{(\alpha+\epsilon) q+\beta(q)\}=\inf _{q \leq \tilde{q}(\alpha)}\{(\alpha+\epsilon) q+\beta(q)\},
$$

and so $\tilde{q}(\alpha+\epsilon) \leq \tilde{q}(\alpha)$.

Note that $-\alpha^{\circ}$ is the one-sided derivative of $\beta$ at 0 , which exists by convexity. That the supremum is $\beta(0)$ and attained at $\alpha^{\circ}$ when finite is in [33, Theorems 12.2 and 27.1]. Otherwise, $\beta(q)=\infty$ for $q<0$, which means that $\beta^{*}(\alpha)=\inf \{q \alpha+\beta(q): q \geq 0\}$, which is increasing in $\alpha$ and strictly less than $\beta(0)$.

Consider $f(\theta)=m(\theta, q \alpha-\theta \alpha+\phi)$. This is a Laplace transform, and so convex and differentiable when finite, and its first derivative is easily checked to be 0 at $\theta=q$ using the definition of $\alpha$. Hence, $m(\theta, q \alpha-\theta \alpha+\phi) \geq m(q, \phi)=1$, and $\beta(q)=\phi$. Therefore, $\beta(\theta) \geq q \alpha-\theta \alpha+\beta(q)$ for all $\theta$, which implies that $\beta^{*}(\alpha)=q \alpha+\beta(q)$.

\section{Results for general branching processes}

Recall that $-\log p_{i}=z_{i}$ and $-\log r_{i}=\sigma_{i}$. We generalise the BRW $Z$ by allowing the birth time $\sigma_{i}$ of individual $\boldsymbol{i}$ to depend on the birth time of its parent. Specifically, if $|\boldsymbol{i}|=n$ and $i[n]=j$, then $\sigma_{i}=\sigma_{i-}-\log r_{j}(\boldsymbol{i}-)$. An illustration of this process and its encoding of the random self-similar measure is given in Figure 1. 


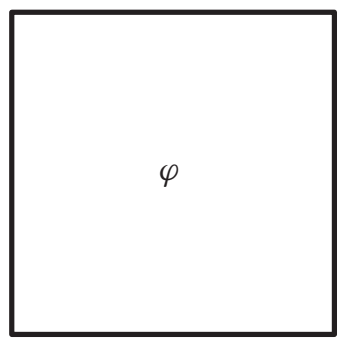

Iteration 0

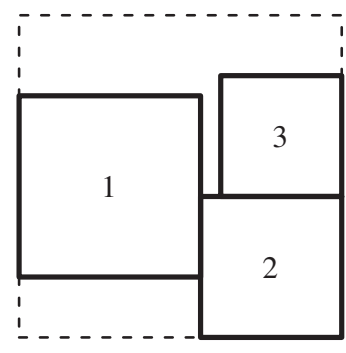

Iteration 1

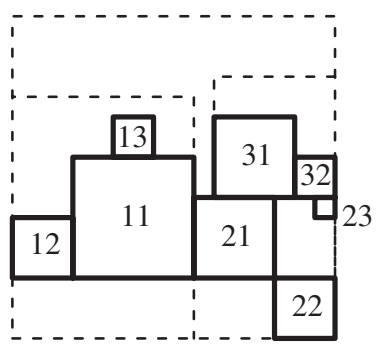

Iteration 2

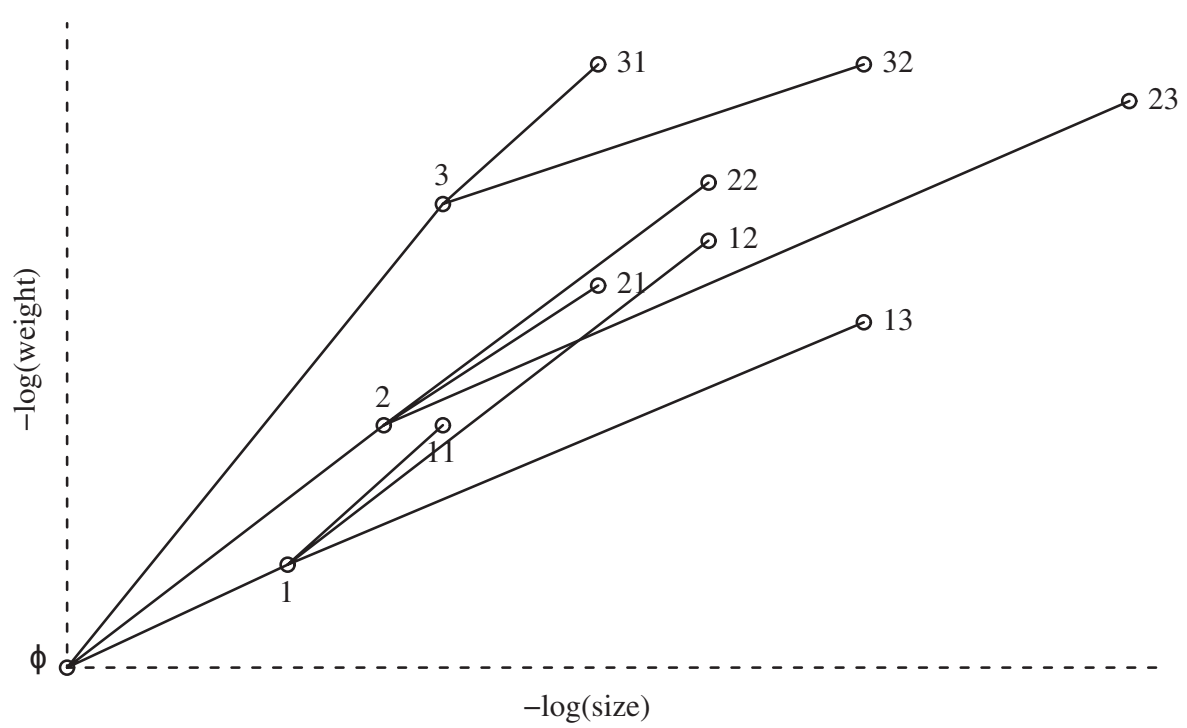

FIGURE 1: At the top we have three iterations in the realisation of a random fractal construction, with components indexed using our tree notation. Here the set $\varphi$ corresponds to $K$, the set 1 to $K_{1}=S_{1}(K)$, and so on. At the bottom we have the corresponding general branching random walk, where sets in iteration $k$ correspond to individuals in generation $k$. The birth time of an individual (along the $x$-axis) is $-\log$ of the diameter of the set, and the birth position is $-\log$ of the weight of the set. An infinite line of descent in the BRW corresponds to a point in the limiting fractal set, and (formally) the asymptotic slope of the trajectory gives the local dimension at that point. It is possible for a point in the fractal to have more than one corresponding line of descent (such points lie on the boundary of two or more sets, for example, $K_{11}$ and $K_{21}$ above). These points are the principle difficulty faced in computing a lower bound for the multifractal spectrum; however, in Section 7 we show that in fact they do not contribute at all.

In the course of the main proofs, estimates will be needed of several quantities with the general form $\mathrm{E} \sum_{\boldsymbol{i} \in \mathcal{Q}} \mathrm{e}^{-q z_{i}-\gamma \sigma_{i}} W_{i}^{q}$ for various sets of individuals $\mathcal{Q}$. In this section we develop these estimates, by drawing on branching process theory. It is worth pointing out that the full strength of that theory, as exhibited in the convergence results in [28], is not employed. The calculations are eased by introducing an auxiliary probability measure, $\widetilde{\mathrm{P}}$, which is done next. Its importance arises from the simple and well-known lemma following the definition. A first application of this lemma is then given. 
Definition 4.1. When $q$ and $\beta$ are such that

$$
m(q, \beta)=\mathrm{E} \sum_{i \in T_{1}} \mathrm{e}^{-q z_{i}-\beta \sigma_{i}} \leq 1,
$$

define the probability measure $\eta=\eta_{q, \beta}$ by

$$
\eta(A)=\mathrm{E} \sum_{i \in T_{1}} \mathrm{e}^{-q z_{i}-\beta \sigma_{i}} I\left(\left(z_{i}, \sigma_{i}\right) \in A\right)
$$

for any Borel set $A \subset \mathbb{R}^{2}$ and $\eta(\infty, \infty)=1-\eta\left(\mathbb{R}^{2}\right)$. Hence, $\eta$ is a probability measure on $\mathbb{R}^{2}$ when $m(q, \beta)=1$. Let $\left(R_{n}, S_{n}\right)$ denote the random walk with independent identical increments chosen according to $\eta$ with initial state $\left(R_{0}, S_{0}\right)=(0,0)$. We use $\widetilde{\mathrm{E}}$ and $\widetilde{\mathrm{P}}$ for the expectation and probability of this random walk. Note that $\widetilde{\mathrm{P}}\left(S_{1}>0\right)=1$, because $\sigma_{i}=-\log r_{i}>0$ for any $i \in T_{1}$.

Lemma 4.1. For $(q, \beta)$ satisfying (4.1) and any positive function $g$, with $g(\infty)=0$ in the case when $m(q, \beta)<1$,

$$
\mathrm{E} \sum_{i \in T_{n}} p_{i}^{q} r_{i}^{\beta} g\left(-\log r_{i}\right)=\mathrm{E} \sum_{i \in T_{n}} \mathrm{e}^{-q z_{i}-\beta \sigma_{i}} g\left(\sigma_{i}\right)=\widetilde{\mathrm{E}} g\left(S_{n}\right) .
$$

Proof. The proof can be found in, for example, [9, Lemma 4.1]: the case where $m(q, \beta)<1$ is just a minor reformulation.

Lemma 4.2. If $m(q, \beta) \leq 1$ then, for $\epsilon>0, \widetilde{\mathrm{E}}^{-\epsilon S_{1}}<1$ and

$$
\mathrm{E} \sum_{i \in T_{n}} \mathrm{e}^{-q z_{i}-\beta \sigma_{i}} \mathrm{e}^{-\epsilon \sigma_{i}} W_{i}^{q}=\left(\widetilde{\mathrm{E}} \mathrm{e}^{-\epsilon S_{1}}\right)^{n} \mathrm{E} W^{q}
$$

Proof. Note first that $\widetilde{\mathrm{E}} \mathrm{e}^{-\epsilon S_{1}}<1$, since $\widetilde{\mathrm{P}}\left(S_{1}>0\right)=1$. Also, using Lemma 4.1 for the second equality,

$$
\mathrm{EE}\left[\sum_{i \in T_{n}} \mathrm{e}^{-q z_{i}-\beta \sigma_{i}} \mathrm{e}^{-\epsilon \sigma_{i}} W_{i}^{q} \mid \mathcal{B}_{n}\right]=\left(\mathrm{E} W^{q}\right) \mathrm{E} \sum_{i \in T_{n}} \mathrm{e}^{-q z_{i}-\beta \sigma_{i}} \mathrm{e}^{-\epsilon \sigma_{i}}=\left(\mathrm{E} W^{q}\right) \widetilde{\mathrm{E}} \mathrm{e}^{-\epsilon S_{n}},
$$

which gives the result.

Suppose that $\psi: \Omega \times \mathbb{R} \rightarrow[0, \infty)$, so $\psi$ is a function from $\mathbb{R}$ to $[0, \infty)$ that is random, in the sense that it is defined on a random marked tree. Let $\Psi$ be its expectation. Such a function is usually called a random characteristic in the branching process literature. Let $\psi_{i}$ be the corresponding function defined on the subtree rooted at $i$. Recall that $\mathscr{B}_{1}$ is the $\sigma$-algebra containing all information on the life story of the initial ancestor and that $\mathcal{B}_{n}$ is the information on the life histories of the first $n-1$ generations. The remaining estimates use the idea of a random characteristic and rely on renewal theory applied to $\left(S_{n}\right)$. The key result is given next. The defective case, which is easier, could be discussed separately-to encompass it here, define $\Psi(-\infty)=0$.

Lemma 4.3. Assume that $m(q, \beta) \leq 1$ and that

$$
\sum_{n=-\infty}^{\infty} \sup \{\Psi(t): n \leq t<n+1\}<\infty
$$


Then there is a finite $C$ such that, for all $t>0$,

$$
\mathrm{E} \sum_{n} \sum_{i \in T_{n}} \mathrm{e}^{-q z_{i}-\beta \sigma_{i}} \psi_{i}\left(t-\sigma_{i}\right) \leq C .
$$

Proof. Note first, again using Lemma 4.1 for the second equality, that

$$
\mathrm{E} \sum_{i \in T_{n}} \mathrm{e}^{-q z_{i}-\beta \sigma_{i}} \psi_{\boldsymbol{i}}\left(t-\sigma_{i}\right)=\mathrm{E} \sum_{i \in T_{n}} \mathrm{e}^{-q z_{i}-\beta \sigma_{i}} \Psi\left(t-\sigma_{i}\right)=\widetilde{\mathrm{E}} \Psi\left(t-S_{n}\right) .
$$

Thus, with $U$ the renewal measure associated with $\left(S_{n}\right)$,

$$
\mathrm{E} \sum_{n} \sum_{i \in T_{n}} \mathrm{e}^{-q z_{i}-\beta \sigma_{i}} \psi_{i}\left(t-\sigma_{i}\right)=\int \Psi(t-\sigma) U(\mathrm{~d} \sigma),
$$

which is bounded when (4.2) holds by the renewal theorem.

We will need to consider certain special sets of nodes which we now introduce. Recall that $\boldsymbol{i}-$ is the parent of $\boldsymbol{i}$, and let

$$
\mathcal{C}(t)=\left\{i \in I: \sigma_{i-} \leq t<\sigma_{i}\right\} .
$$

The collection $\mathcal{C}(t)$ is critical in the study of general branching process [18], [28], and is often called the coming generation at time $t$ in that literature.

Lemma 4.4. If $m(q, \beta) \leq 1, \beta>0$, and

$$
\mathrm{E} \sum_{i \in T_{1}} \mathrm{e}^{-q z_{i}}<\infty
$$

then there is a finite $C$ such that, for all $t>0$,

$$
\mathrm{Ee}^{-\beta t} \sum_{i \in \mathcal{C}(t)} \mathrm{e}^{-q z_{i}} W_{i}^{q} \leq C \mathrm{E} W^{q} .
$$

Proof. Assume that $\mathrm{E} W^{q}<\infty$. Let

$$
\psi(t)=\mathrm{e}^{-\beta t} \sum_{j} \mathrm{e}^{-q z_{j}} W_{j}^{q} I\left(0 \leq t<\sigma_{j}\right) .
$$

Then it is straightforward to check that

$$
\sum_{i \in T} \mathrm{e}^{-q z_{i}-\beta \sigma_{i}} \psi_{\boldsymbol{i}}\left(t-\sigma_{\boldsymbol{i}}\right)=\mathrm{e}^{-\beta t} \sum_{\boldsymbol{i} \in \mathcal{C}(t)} \mathrm{e}^{-q z_{i}} W_{i}^{q} .
$$

Furthermore, because $\beta>0$,

$$
\begin{aligned}
\sum_{n} \sup _{n \leq t<n+1} \Psi(t) & \leq \mathrm{E} \sum_{j} \mathrm{e}^{-q z_{j}} W_{j}^{q} \sum_{n} \sup _{n \leq t<n+1} \mathrm{e}^{-\beta t} I\left(0 \leq t<\sigma_{j}\right) \\
& \leq \frac{1}{1-\mathrm{e}^{-\beta}} \mathrm{E} \sum_{j} \mathrm{e}^{-q z_{j}} \mathrm{E} W^{q},
\end{aligned}
$$

which is finite by assumption. Now, Lemma 4.3 gives the result. 
We need another collection of individuals: let $g(t)$ be the first in their line of descent with a child in $\mathcal{C}(t)$. This is the 'lower boundary' of the set of parents of those in $\mathcal{C}(t)$. More formally, let $L_{i}=\max \left\{\sigma_{i j}-\sigma_{i}: j\right\}$, which is the age of $i$ at the birth of her last child. Now let

$$
\mathscr{L}(t)=\left\{\boldsymbol{i}: \sigma_{i}+L_{i}>t \text { and } \sigma_{j}+L_{j} \leq t \text { for all } \boldsymbol{j}<\boldsymbol{i}\right\},
$$

so that, in particular, $g(t)$ is just the initial ancestor for $0 \leq t<L_{\varnothing}$. Before giving the main lemma, we need a preparatory lemma that simplifies moment conditions.

Lemma 4.5. For $q \geq 1$ and a positive function $g, \mathrm{E} g(L) W^{q}$ is finite when $\mathrm{E} W^{q}<\infty$ and

$$
\mathrm{E}\left[g(L)\left(\sum_{j} \mathrm{e}^{-z_{j}}\right)^{q}\right]<\infty .
$$

Proof. Rewriting, using (2.1),

$$
g(L) W^{q}=g(L)\left(\sum_{j} \mathrm{e}^{-z_{j}} W_{j}\right)^{q},
$$

where, given $\mathscr{B}_{1}$, the $W_{j}$ are independent and distributed as $W$. Now, by a variation on Rosenthal's inequality [20, Corollary 3], and then the fact that $\mathrm{E} W=1$,

$$
\begin{aligned}
\mathrm{E}\left[\left(\sum_{j} \mathrm{e}^{-z_{j}} W_{j}\right)^{q} \mid \mathscr{B}_{1}\right] & \leq C \max \left\{\left(\sum_{j} \mathrm{e}^{-z_{j}} \mathrm{E} W\right)^{q},\left(\sum_{j} \mathrm{e}^{-q z_{j}}\right) \mathrm{E} W^{q}\right\} \\
& \leq C\left(\sum_{j} \mathrm{e}^{-z_{j}}\right)^{q} \mathrm{E} W^{q} .
\end{aligned}
$$

Hence,

$$
\mathrm{E} g(L) W^{q} \leq C \mathrm{E}\left[g(L)\left(\sum_{j} \mathrm{e}^{-z_{j}}\right)^{q}\right] \mathrm{E} W^{q},
$$

which is finite under the assumptions.

Lemma 4.6. Assume that $m(q, \beta) \leq 1$ and $q>1$. Then, if $\gamma-\beta>0$ and (4.4) holds with $g(x)=\mathrm{e}^{(\gamma-\beta) x}$, there is a finite $C$ such that, for all $t>0$,

$$
\mathrm{Ee}^{(\gamma-\beta) t} \sum_{\boldsymbol{j} \in \mathcal{g}(t)} \mathrm{e}^{-q z_{j}-\gamma \sigma_{j}} W_{j}^{q} \leq C \mathrm{E} W^{q} .
$$

Proof. Note that

$$
\mathscr{g}(t) \subset \mathcal{P}(t)=\left\{i: \sigma_{i} \leq t<\sigma_{i}+L_{i}\right\},
$$

where $\mathcal{P}(t)$ is just the set of parents of $\mathcal{C}(t)$. Hence, it is sufficient, and less complicated, to bound

$$
\mathrm{Ee}^{(\gamma-\beta) t} \sum_{j \in \mathcal{P}(t)} \mathrm{e}^{-q z_{j}-\gamma \sigma_{j}} W_{j}^{q} .
$$

We can assume that $\mathrm{E} W^{q}<\infty$, for the result is obviously true otherwise. Let

$$
\psi(t)=\mathrm{e}^{(\gamma-\beta) t} W^{q} I(0 \leq t<L) .
$$


A straightforward calculation shows that

$$
\sum_{\boldsymbol{j} \in T} \mathrm{e}^{-q z_{j}-\beta \sigma_{j}} \psi_{\boldsymbol{j}}\left(t-\sigma_{j}\right)=\mathrm{e}^{(\gamma-\beta) t} \sum_{\boldsymbol{j} \in \mathcal{P}(t)} \mathrm{e}^{-q z_{j}-\gamma \sigma_{j}} W_{j}^{q} .
$$

Using $\Psi(t)=0$ for all $t \leq 0$ and $\gamma-\beta>0$,

$$
\begin{aligned}
\sum_{n=-\infty}^{\infty} \sup _{n \leq t<n+1} \Psi(t) & =\sum_{n=0}^{\infty} \sup _{n \leq t<n+1} \Psi(t) \\
& \leq \sum_{n=0}^{\infty} \mathrm{E}\left[W^{q} \sup _{n \leq t<n+1}\left\{\mathrm{e}^{(\gamma-\beta) t} I(0 \leq t<L)\right\}\right] \\
& \leq \sum_{n=0}^{\infty} \mathrm{E}\left[W^{q} \mathrm{e}^{(\gamma-\beta)(n+1)} I(n<L)\right] \\
& \leq \frac{\mathrm{e}^{2(\gamma-\beta)}}{\mathrm{e}^{\gamma-\beta}-1} \mathrm{E} W^{q} \mathrm{e}^{(\gamma-\beta) L} .
\end{aligned}
$$

By Lemma 4.5, this is finite. Hence, (4.2) holds and so Lemma 4.3 applies to give the result.

Note that, when $g(x)=\mathrm{e}^{(\gamma-\beta) x}$ with $\gamma-\beta>0$, (4.4) can hold only if $L=L_{\varnothing}$ is always finite, which implies that $\mathrm{P}(N<\infty)=1$.

\section{Upper bound on $\operatorname{dim}_{H}\left(F_{\alpha}\right)$}

Lemma 2.4 bounds $\mu_{\pi}(B)$ using $T_{n}$. The next lemma provides a couple of variants of this bound, using $\mathcal{C}(t)$ and $\mathcal{g}(t)$.

Lemma 5.1. We have

$$
\mu_{\pi}(B) \leq \sum_{j \in \mathcal{C}(t)} p_{j} W_{j} I\left(B \cap K_{j} \neq \varnothing\right), \quad \mu_{\pi}(B) \leq \sum_{j \in \mathcal{g}(t)} p_{j} W_{j} I\left(B \cap K_{j} \neq \varnothing\right) .
$$

Proof. Much as the proof of Lemma 2.4,

$$
\mu_{\pi}(B) \leq \sum_{|\boldsymbol{j}| \leq l, \boldsymbol{j} \in \mathcal{C}(t)} \mu\left(C_{\boldsymbol{j}}\right) I\left(B \cap K_{\boldsymbol{j}} \neq \varnothing\right)+\sum_{\boldsymbol{j} \in T_{l}, \sigma_{j} \leq t} \mu\left(C_{\boldsymbol{j}}\right) I\left(B \cap K_{\boldsymbol{j}} \neq \varnothing\right),
$$

but, by Lemma 2.1, $\inf \left\{\sigma_{j}: j \in T_{l}\right\}$ goes to $\infty$ with $l$ (P-a.s.) and so the second sum here is eventually empty. Similarly,

$$
\mu_{\pi}(B) \leq \sum_{|\boldsymbol{j}| \leq l, \boldsymbol{j} \in \mathcal{g}(t)} \mu\left(C_{\boldsymbol{j}}\right) I\left(B \cap K_{\boldsymbol{j}} \neq \varnothing\right)+\sum_{\boldsymbol{j} \in T_{l}, \boldsymbol{j}<\mathcal{g}(t)} \mu\left(C_{\boldsymbol{j}}\right) I\left(B \cap K_{\boldsymbol{j}} \neq \varnothing\right)
$$

and

$$
\left\{\boldsymbol{j}<\mathcal{g}(t): \boldsymbol{j} \in T_{l}\right\} \subset\left\{\left(\sigma_{j}+L_{j}\right) \leq t: \boldsymbol{j} \in T_{l}\right\} \subset\left\{\sigma_{j} \leq t: \boldsymbol{j} \in T_{l}\right\},
$$

and, by Lemma 2.1 again, the final set is eventually empty for large $l$.

We now state four upper-bound lemmas (that between them cover all cases) and the upper bound that results from combining them before giving the proofs of these lemmas. The open set 
condition and that $K$ has a $\gamma$-boundary are needed only in the fourth of the lemmas, Lemma 5.5, where $q>1$. The basic idea is to fix on a $(q, \beta)$ such that $m(q, \beta) \leq 1$ and use this to bound the Hausdorff dimension of $F_{\alpha}$ for any $\alpha$. Then we can vary $(q, \beta)$ to produce the best bound for $\operatorname{dim}_{H}\left(F_{\alpha}\right)$ these results entail. It will be useful to work with some sets that contain $F_{\alpha}$ but have some monotonicity in $\alpha$. Let

$$
\begin{aligned}
& \underline{F_{\alpha}}=\left\{x \in F: \liminf _{r \rightarrow 0} \frac{\log \mu_{\pi}(B(x ; r))}{\log r} \geq \alpha\right\}, \\
& \overline{F_{\alpha}}=\left\{x \in F: \limsup _{r \rightarrow 0} \frac{\log \mu_{\pi}(B(x ; r))}{\log r} \leq \alpha\right\} .
\end{aligned}
$$

Lemma 5.2. Suppose that Assumption A holds. Let $q \leq 0$. Suppose that

$$
(q, \beta) \text { are such that } m(q, \beta)=\mathrm{E} \sum_{i} p_{i}^{q} r_{i}^{\beta} \leq 1
$$

and

$$
\mathrm{E} W^{q}<\infty
$$

Then $\operatorname{dim}_{H}\left(\underline{F_{\alpha}}\right) \leq q \alpha+\beta$ a.s. when $q \alpha+\beta \geq 0$, and $\underline{F_{\alpha}}=\varnothing$ a.s. when $q \alpha+\beta<0$.

Lemma 5.3. (cf. Theorem 1.3 of [27].) When Assumption A(iii) holds, $\operatorname{dim}_{H}(F) \leq \beta(0)$.

Lemma 5.4. Suppose that Assumption A holds. Let $0<q \leq 1$. Suppose that (5.1) holds and

$$
m(q, 0)=\mathrm{E} \sum_{i} p_{i}^{q}<\infty .
$$

Then $\operatorname{dim}_{H}\left(\overline{F_{\alpha}}\right) \leq q \alpha+\beta$ a.s. when $q \alpha+\beta \geq 0$, and $\overline{F_{\alpha}}=\varnothing$ a.s. when $q \alpha+\beta<0$.

Lemma 5.5. Suppose that Assumption A holds. Let $q>1$. Suppose that (5.1) and (5.2) hold. Suppose also that the open set condition holds, that $K$ has a $\gamma$-boundary, and

$$
\mathrm{E}\left[\left(\min _{i} r_{i}\right)^{\beta-\gamma}\left(\sum_{i} p_{i}\right)^{q}\right]<\infty .
$$

Then $\operatorname{dim}_{H}\left(\overline{F_{\alpha}}\right) \leq q \alpha+\beta$ a.s. when $q \alpha+\beta \geq 0$, and $\overline{F_{\alpha}}=\varnothing$ a.s. when $q \alpha+\beta<0$.

Later, in Section 9, conditions for (5.2) are discussed. It is worth noting that (5.4) is a strengthening of part of the condition (9.1) needed to give (5.2) when $q>1$.

These four lemmas combine to give the next two propositions, which are just the upper bound in Theorem 3.1, with a couple of the conditions moved to where they bite. It focuses on a single $\alpha$ and, effectively, takes the $(q, \beta)$ to make $q \alpha+\beta$ as small as it can to be consistent with $m(q, \beta) \leq 1$. In the excluded case, where $\left(D_{L}, D_{U}\right)$ is empty, by Lemma 3.2(v), there is a $\gamma$ such that $\beta^{*}(a)=-\infty$ unless $a=\gamma$ when $\beta^{*}(\gamma)=\gamma$ and then $F_{\alpha}$ is empty unless $a=\gamma$ and $\operatorname{dim}_{H}\left(F_{\gamma}\right) \leq \operatorname{dim}_{H}(F) \leq \gamma$.

Proposition 5.1. Suppose that Assumption A holds. Let $\alpha \in\left(D_{L}, D_{U}\right)$, with $q=\tilde{q}(\alpha)$ and $\beta=\beta(\tilde{q}(\alpha))$ as in Lemma 3.2(vi). Assume that (5.2) holds. If $0<\tilde{q}(\alpha) \leq 1$, assume that (5.3) holds. If $\tilde{q}(\alpha)>1$, assume that the open set condition holds, that $K$ has a $\gamma$-boundary, and that (5.4) holds. Then, if $\beta^{*}(\alpha) \geq 0, \operatorname{dim}_{H}\left(F_{\alpha}\right) \leq \beta^{*}(\alpha)$, P-a.s. 
Proof. By Lemma 3.2(vi), $\beta^{*}(\alpha)=\alpha \tilde{q}(\alpha)+\beta(\tilde{q}(\alpha))=\alpha q+\beta$ and, by Lemma 3.2(iv), $m(\tilde{q}(\alpha), \beta(\tilde{q}(\alpha))) \leq 1$. The rest of the conditions ensure that the appropriate one of the four lemmas applies.

Proposition 5.2. Under the same conditions as Proposition 5.1, if $\beta^{*}(\alpha)<0$ then (a.s.), when $\alpha<\alpha^{\circ}, F_{\beta}=\varnothing$ for all $\beta \leq \alpha$, and when $\alpha^{\circ}<\infty$ and $\alpha>\alpha^{\circ}, F_{\beta}=\varnothing$ for all $\beta \geq \alpha$.

Proof. For $\beta<\alpha, F_{\beta} \subset \overline{F_{\beta}} \subset \overline{F_{\alpha}}$. For $\alpha<\alpha^{\circ}, \tilde{q}(\alpha)>0$ and then Lemmas 5.4 and 5.5 imply that $\overline{F_{\alpha}}=\varnothing$. Similarly, for $\alpha>\alpha^{\circ}, \tilde{q}(\alpha)<0$, Lemma 5.2 implies that $\underline{F_{\alpha}}=\varnothing$ and, for $\beta>\alpha, F_{\beta} \subset \underline{F_{\beta}} \subset \underline{F_{\alpha}}$.

Our approach to all four proofs has similarities to that employed in [2, Lemma 4.2]. Let $\mathscr{H}^{d}(A)$ be the Hausdorff $d$-dimensional measure of the set $A$, and let

$$
\mathcal{H}_{\delta}^{\kappa}(A)=\inf \left\{\sum_{i}\left|B_{i}\right|^{\kappa}: A \subset \bigcup_{i} B_{i},\left|B_{i}\right| \leq \delta\right\},
$$

where the $B_{i}$ are balls. Then

$$
\mathscr{H}^{\kappa}(A)=\lim _{\delta \downarrow 0} \mathscr{H}_{\delta}^{\kappa}(A) \quad \text { and } \quad \operatorname{dim}_{H}(A)=\inf \left\{\kappa: \mathscr{H}^{\kappa}(A)=0\right\} .
$$

Proof of Lemma 5.2. Let

$$
r(k)=\sup \left\{r_{i \mid n}: n \geq k, i \in \partial T\right\},
$$

which goes to 0 as $k$ goes to $\infty$ by Lemma 2.1. For each $\epsilon \in(0, \alpha), c>1$, and $k \in \mathbb{N}$, the collection

$$
\mathcal{V}_{\epsilon, c, k}=\left\{B\left(\pi(\boldsymbol{i}) ; c r_{\boldsymbol{i} \mid n}\right): \boldsymbol{i} \in \partial T, n \geq k, \mu_{\pi}\left(B\left(\pi(\boldsymbol{i}) ; c r_{\boldsymbol{i} \mid n}\right)\right) \leq\left(2 c r_{\boldsymbol{i} \mid n}\right)^{(\alpha-\epsilon)}\right\}
$$

is a Vitali class for $\underline{F_{\alpha}}$ (that is, a collection from which, for any $\delta>0$, a covering can be selected with every member having diameter less than $\delta$ ). By a Vitali covering lemma, a collection of disjoint members of $\mathcal{V}_{\epsilon, c, k}$ can be selected such that, when their radii are multiplied by 5 , they cover $\underline{F_{\alpha}}$. Let $\left(B_{i}\right)$ be such a disjoint collection, which we call a Vitali collection. Then

$$
\mathcal{H}_{5 c r(k)}^{\kappa} \underline{\left(F_{\alpha}\right)} \leq 5^{\kappa} \sum_{i}\left|B_{i}\right|^{\kappa} .
$$

The idea is to show that, for suitable $\kappa$, the right-hand side here is bounded a.s. by a variable going to 0 as $k \uparrow \infty$. Then $\operatorname{dim}_{H}\left(\underline{F_{\alpha}}\right) \leq \kappa$.

Since $c>1, K_{i \mid n} \subset B\left(\pi(\boldsymbol{i}) ; c \overline{r_{i \mid n}}\right)$, and then

$$
\mu_{\pi}\left(B\left(\pi(\boldsymbol{i}) ; c r_{\boldsymbol{i} \mid n}\right)\right) \geq \mu_{\pi}\left(K_{\boldsymbol{i} \mid n}\right) \geq \mu\left(C_{\boldsymbol{i} \mid n}\right)=p_{\boldsymbol{i} \mid n} W_{\boldsymbol{i} \mid n} .
$$

Hence, for $B\left(\pi(i) ; c r_{i \mid n}\right) \in \mathcal{V}_{\epsilon, c, k}$ and $q \leq 0$,

$$
\left|B\left(\pi(\boldsymbol{i}) ; c r_{\boldsymbol{i} \mid n}\right)\right|^{(\alpha-\epsilon) q}=\left(2 c r_{\boldsymbol{i} \mid n}\right)^{(\alpha-\epsilon) q} \leq \mu_{\pi}\left(B\left(\pi(\boldsymbol{i}) ; c r_{\boldsymbol{i} \mid n}\right)\right)^{q} \leq\left(p_{\boldsymbol{i} \mid n} W_{\boldsymbol{i} \mid n}\right)^{q},
$$

and so

$$
\left|B\left(\pi(\boldsymbol{i}) ; c r_{\boldsymbol{i} \mid n}\right)\right|^{(\alpha-\epsilon) q+\beta+\epsilon} \leq\left(p_{\boldsymbol{i} \mid n} W_{\boldsymbol{i} \mid n}\right)^{q}\left(2 c r_{\boldsymbol{i} \mid n}\right)^{\beta+\epsilon} .
$$


Then, using disjointness of the $\left(B_{i}\right)$ to deduce that each term on the right-hand side occurs at most once,

$$
\sum_{i}\left|B_{i}\right|^{(\alpha-\epsilon) q+\beta+\epsilon} \leq \sum_{n=k}^{\infty} \sum_{\boldsymbol{j} \in T_{n}}\left(p_{j} W_{j}\right)^{q}\left(2 c r_{j}\right)^{\beta+\epsilon} .
$$

Let the expression on the right-hand side here be $U_{k}$; it follows from Lemma 4.2 that this goes to 0 a.s. as $k \uparrow \infty$. Hence, using this and (5.5), $\mathscr{H}^{(\alpha-\epsilon) q+\beta+\epsilon}\left(\underline{F_{\alpha}}\right)=0$, giving $\operatorname{dim}_{H}\left(\underline{F_{\alpha}}\right) \leq \alpha q+\beta$.

Now suppose that $q \alpha+\beta<0$, so that, for suitably small $\epsilon>0, \alpha q+\beta+\epsilon|q|+\epsilon<0$. Taking a Vitalli collection from $\mathcal{V}_{\epsilon, c, k}$,

$$
\sum_{i}\left|B_{i}\right|^{\alpha q+\beta+\epsilon|q|+\epsilon} \leq U_{k} \rightarrow 0
$$

as $k \uparrow \infty$. Every term on the left-hand side exceeds $(2 c)^{\alpha q+\beta+\epsilon|q|+\epsilon}$, and so, for large enough $k$, the sum must be empty. Thus, $\mathcal{V}_{\epsilon, c, k}$ must be empty, which implies that $\underline{F_{\alpha}}$ is empty.

Proof of Lemma 5.3. The collection

$$
\mathcal{V}_{k}=\left\{B\left(\pi(\boldsymbol{i}) ; r_{i \mid n}\right): i \in \partial T, n \in \mathbb{N}, n \geq k\right\}
$$

is a Vitali class for $F$. Let $\left(B_{i}\right)$ be a Vitali covering from $\mathcal{V}_{k}$. Take $\beta=\beta(0)$. Much as in the previous proof,

$$
\sum_{i}\left|B_{i}\right|^{\beta+\epsilon} \leq \sum_{n=k}^{\infty} \sum_{j \in T_{n}}\left(2 r_{j}\right)^{\beta+\epsilon}
$$

and Lemma 4.2 applies to this with $q=0$. The proof that $\operatorname{dim}_{H}(F) \leq \beta(0)$ now mimics that of $\operatorname{dim}_{H}\left(\underline{F_{\alpha}}\right) \leq \alpha q+\beta$ in Lemma 5.2.

Proof of Lemma 5.4. For each $\epsilon \in(0, \alpha), \rho \in(0,1)$, and $k \in \mathbb{N}$, the collection

$$
\mathcal{V}_{\epsilon, \rho, k}^{\prime}=\left\{B\left(\pi(\boldsymbol{i}) ; \frac{\rho^{n}}{2}\right): i \in \partial T, n \in \mathbb{N}, n \geq k, \rho^{n(\alpha+\epsilon)} \leq \mu_{\pi}\left(B\left(\pi(\boldsymbol{i}) \frac{\rho^{n}}{2}\right)\right)\right\}
$$

is a Vitali class for $\overline{F_{\alpha}}$. Let $\left(B_{i, n}\right)$ be a Vitali collection from $\mathcal{V}_{\epsilon, \rho, k}^{\prime}$ in which $B_{i, n}$ has diameter $\rho^{n}$. Then

$$
\mathscr{H}_{5 \rho^{k}}^{\kappa}\left(\overline{F_{\alpha}}\right) \leq 5^{\kappa} \sum_{n=k}^{\infty} \sum_{i}\left|B_{i, n}\right|^{\kappa} .
$$

It will be convenient to let $\mathcal{C}_{n}=\mathcal{C}(-n \log \rho)$. Take $B \in \mathcal{V}_{\epsilon, \rho, k}^{\prime}$. Since $0<q \leq 1$, using Lemma 5.1,

$$
\mu_{\pi}(B)^{q} \leq \sum_{j \in \mathcal{C}_{n}}\left(p_{j} W_{j}\right)^{q} I\left(B \cap K_{j} \neq \varnothing\right),
$$

and so

$$
\left|B_{i, n}\right|^{(\alpha+\epsilon) q}=\left(\rho^{n(\alpha+\epsilon)}\right)^{q} \leq \mu_{\pi}\left(B_{i, n}\right)^{q} \leq \sum_{\boldsymbol{j} \in \mathcal{C}_{n}}\left(p_{j} W_{j}\right)^{q} I\left(B_{i, n} \cap K_{j} \neq \varnothing\right) .
$$

Let $\mathfrak{n}$ be the maximum number of disjoint balls of diameter greater than 1 that can overlap with $K ; \mathfrak{n}$ is finite because $K$ is bounded. Hence, for $\boldsymbol{j} \in \mathcal{C}_{n}$, there are at most $\mathfrak{n}$ disjoint balls of diameter $\rho^{n}$ (in $\mathcal{V}_{\epsilon, \rho, k}^{\prime}$ ) that overlap with $K_{j}$. Therefore,

$$
\sum_{i}\left|B_{i, n}\right|^{(\alpha+\epsilon) q+\beta+\epsilon}=\sum_{i}\left|B_{i, n}\right|^{(\alpha+\epsilon) q} \rho^{n(\beta+\epsilon)} \leq \rho^{n \epsilon} \mathfrak{n} \sum_{j \in \mathcal{C}_{n}}\left(p_{j} W_{j}\right)^{q} \rho^{n \beta},
$$


and so

$$
\sum_{n=k}^{\infty} \sum_{i}\left|B_{i, n}\right|^{(\alpha+\epsilon) q+\beta+\epsilon} \leq \mathfrak{n} \sum_{n=k}^{\infty} \rho^{n \epsilon} \sum_{j \in \mathfrak{C}_{n}}\left(p_{\boldsymbol{j}} W_{\boldsymbol{j}}\right)^{q} \rho^{n \beta} .
$$

Let $U_{k}$ be defined such that the right-hand side of (5.7) is $\mathfrak{n} U_{k}$. Taking expectations, using Lemma 4.4, gives

$$
\mathrm{E} U_{k}=\mathrm{E}\left[\sum_{n=k}^{\infty} \rho^{n \epsilon} \sum_{\boldsymbol{j} \in \mathcal{C}_{n}}\left(p_{\boldsymbol{j}} W_{\boldsymbol{j}}\right)^{q} \rho^{n \beta}\right] \leq C \mathrm{E}\left[W^{q}\right] \sum_{n=k}^{\infty} \rho^{n \epsilon}=\frac{C \mathrm{E}\left[W^{q}\right] \rho^{k \epsilon}}{1-\rho^{\epsilon}},
$$

and so

$$
U_{k} \rightarrow 0 \quad \text { a.s. as } k \rightarrow \infty .
$$

Hence, using this, (5.6), and (5.7), $\mathscr{H}^{(\alpha-\epsilon) q+\beta+\epsilon}\left(\overline{F_{\alpha}}\right)=0$, giving $\operatorname{dim}_{H}\left(\overline{F_{\alpha}}\right) \leq \alpha q+\beta$. The argument is completed as in Lemma 5.2.

Proof of Lemma 5.5. Under the open set condition, a volume argument shows that there is a finite maximum number, $\tilde{\mathfrak{n}}$, of nonoverlapping sets of shape int $K$ and diameter greater than 1 that can intersect with the ball of diameter 1 . This time the idea is to approximate the measure of balls with diameter $\rho^{n}$ using sets $K_{\boldsymbol{j}}$ with diameter larger than $\rho^{n}$, but as little larger as possible. There can be no more than $\tilde{\mathfrak{n}}$ such sets with disjoint interiors covering such a ball. This finite bound on the number of terms will allow the $p$ th powers of sums to be bounded by the sum of $p$ th powers.

Use the Vitali class $\mathcal{V}_{\epsilon, \rho, k}^{\prime}$ for $\overline{F_{\alpha}}$ and select a Vitali collection $\left(B_{i, n}\right)$ from it as in the proof of Lemma 5.4. Let $\mathscr{g}_{n}=\mathscr{g}(-n \log \rho)$, where $\mathcal{g}$ was introduced in (4.3). In general, using Lemma 5.1,

$$
\left|B_{i, n}\right|^{(\alpha+\epsilon) q} \leq \mu_{\pi}\left(B_{i, n}\right)^{q} \leq\left(\sum_{\boldsymbol{j} \in \mathscr{g}_{n}}\left(p_{\boldsymbol{j}} W_{\boldsymbol{j}}\right) I\left(B_{i, n} \cap K_{\boldsymbol{j}} \neq \varnothing\right)\right)^{q} .
$$

There can be no more than $\tilde{\mathfrak{n}}$ terms in the sum here and so

$$
\left|B_{i, n}\right|^{(\alpha+\epsilon) q} \leq \tilde{\mathfrak{n}}^{q-1} \sum_{j \in \mathcal{g}_{n}} p_{j}^{q} W_{j}^{q} I\left(B_{i, n} \cap K_{j} \neq \varnothing\right) .
$$

Let $\xi_{i, n, i}$ be the indicator that $B_{i, n} \subset \operatorname{int}\left(K_{i}\right)$, and let

$$
\xi_{i, n}^{\prime}=1-\sum_{i \in \mathscr{g}_{n}} \xi_{i, n, i}
$$

which is the indicator that $B_{i, n}$ intersects with the boundary of $K_{i}$ for some $i \in g_{n}$. Then, for any $\kappa$,

$$
\sum_{i}\left|B_{i, n}\right|^{\kappa}=\sum_{i}\left|B_{i, n}\right|^{\kappa} \xi_{i, n}^{\prime}+\sum_{i} \sum_{i \in \mathscr{g}_{n}}\left|B_{i, n}\right|^{\kappa} \xi_{i, n, i} .
$$

The two parts here will be bounded separately.

Since $K_{\boldsymbol{j}}$ has diameter $r_{\boldsymbol{j}}$, its boundary can overlap with at most $\hat{h}\left(r_{\boldsymbol{j}} / \rho^{n}\right)$ disjoint balls of diameter $\rho^{n}$, where $\hat{h}$ was introduced in Definition 3.1. Hence,

$$
\sum_{i}\left|B_{i, n}\right|^{(\alpha+\epsilon) q} \xi_{i, n}^{\prime} \leq \tilde{\mathfrak{n}}^{q-1} \sum_{j \in \mathscr{g}_{n}} p_{j}^{q} W_{j}^{q} \hat{h}\left(\frac{r_{j}}{\rho^{n}}\right) .
$$


Balls with $\xi_{i, n, i}=1$ are all in $\operatorname{int}\left(K_{i}\right)$ and are disjoint. Using this and Lemma 2.6, for a fixed $\boldsymbol{i}$,

$$
\begin{aligned}
\sum_{i}\left|B_{i, n}\right|^{(\alpha+\epsilon) q} \xi_{i, n, i} & \leq \sum_{i} \mu_{\pi}\left(B_{i, n}\right)^{q} \xi_{i, n, i} \\
& \leq\left(\sum_{i} \mu_{\pi}\left(B_{i, n}\right) \xi_{i, n, i}\right)^{q} \\
& \leq\left(\mu_{\pi}\left(\operatorname{int}\left(K_{i}\right)\right)\right)^{q} \\
& \leq\left(p_{i} W_{i}\right)^{q}
\end{aligned}
$$

Hence, since both $\tilde{\mathfrak{n}}^{q-1}$ and $\hat{h}(x)$ always exceed 1 ,

$$
\sum_{i} \sum_{i \in \mathscr{g}_{n}}\left|B_{i, n}\right|^{(\alpha+\epsilon) q} \xi_{i, n, i} \leq \tilde{\mathfrak{n}}^{q-1} \sum_{i \in \mathscr{g}_{n}}\left(p_{\boldsymbol{i}} W_{\boldsymbol{i}}\right)^{q} \hat{h}\left(\frac{r_{j}}{\rho^{n}}\right) .
$$

Therefore, combining these two estimates and using the bound on $\hat{h}$ arising from $K$ having a $\gamma$-boundary,

$$
\begin{aligned}
\sum_{i}\left|B_{i, n}\right|^{(\alpha+\epsilon) q+\beta+\epsilon} & =\rho^{n(\beta+\epsilon)} \sum_{i}\left|B_{i, n}\right|^{(\alpha+\epsilon) q} \\
& \leq 2 \tilde{\mathfrak{n}}^{q-1} \rho^{n(\beta+\epsilon)} \sum_{j \in \mathcal{I}_{n}} p_{j}^{q} W_{j}^{q} \hat{h}\left(\frac{r_{j}}{\rho^{n}}\right) \\
& \leq C^{\prime \prime} \rho^{n \epsilon} \mathrm{e}^{(\gamma-\beta)(-n \log \rho)} \sum_{j \in \mathscr{I}_{n}} \mathrm{e}^{-q z_{j}-\gamma \sigma_{j}} W_{j}^{q},
\end{aligned}
$$

where $C^{\prime \prime}$ is independent of $n$. Now, using Lemma 4.6,

$$
\sum_{n=k}^{\infty} \rho^{n \epsilon} \mathrm{Ee}^{(\gamma-\beta)(-n \log \rho)} \sum_{j \in \mathscr{g}_{n}} \mathrm{e}^{-q z_{j}-\gamma \sigma_{j}} W_{j}^{q} \leq \frac{C \mathrm{E}\left[W^{q}\right] \rho^{k \epsilon}}{1-\rho^{\epsilon}},
$$

and so if we let

$$
U_{k}^{\prime}=C^{\prime \prime} \sum_{n=k}^{\infty} \rho^{n \epsilon} \mathrm{e}^{(\gamma-\beta)(-n \log \rho)} \sum_{j \in \mathscr{g}_{n}} \mathrm{e}^{-q z_{j}-\gamma \sigma_{j}} W_{j}^{q}
$$

then $U_{k}^{\prime} \rightarrow 0$ a.s. as $k \rightarrow \infty$. Finally, since the collection is from $\mathcal{V}_{\epsilon, \rho, k}^{\prime}$,

$$
\sum_{n} \sum_{i}\left|B_{i, n}\right|^{(\alpha+\epsilon) q+\beta+\epsilon}=\sum_{n=k}^{\infty} \sum_{i}\left|B_{i, n}\right|^{(\alpha+\epsilon) q+\beta+\epsilon} \leq U_{k}^{\prime},
$$

and the proof is finished in the same way as in Lemmas 5.2 and 5.4.

\section{Upper-bound theorem with a single null set}

It is natural to want the null set to be independent of $\alpha$. The next result gives some conditions for this. To state it, let $\left(\Gamma_{L}, \Gamma_{U}\right)$ be the interior of the set where $\beta^{*}$ is greater than 0 . The proof is only sketched. It would be easy to give other formulations by considering a subset of the collection of values for $\alpha$ covered here. Before giving the result some further facts about $\beta^{*}$ need to be recorded. 
Lemma 6.1. Make the same assumptions as in Lemma 3.2.

(i) $\left(\Gamma_{L}, \Gamma_{U}\right)$ is nonempty when $\left(D_{L}, D_{U}\right)$ is nonempty.

(ii) $D_{L}=\operatorname{essinf}\left(\inf _{i}\left(\left(\log p_{i}\right) /\left(\log r_{i}\right)\right)\right), D_{U}=\operatorname{ess} \sup \left(\sup _{i}\left(\left(\log p_{i}\right) /\left(\log r_{i}\right)\right)\right)$.

(iii) If, for some $\epsilon>0,\left(\Gamma_{L}-\epsilon, \Gamma_{U}+\epsilon\right) \subset\left(D_{L}, D_{U}\right)$ then $\beta^{*}\left(\Gamma_{U}\right)=\beta^{*}\left(\Gamma_{L}\right)=0, \tilde{q}\left(\Gamma_{U}\right) \leq 0$, and $\tilde{q}\left(\Gamma_{L}\right) \geq 1$.

(iv) Suppose that there is no constant $\gamma$ such that $p_{i} r_{i}^{-\gamma}=1$ for all $i$. Suppose too that, for every $q \in\left(q_{1}, q_{2}\right), m$ is finite in a neighbourhood of $(q, \beta(q))$. Then, on $\left(q_{1}, q_{2}\right)$, $m(q, \beta(q))=1, \beta$ is strictly convex, $\beta^{\prime}(q)$ is strictly increasing,

$$
-\beta^{\prime}(q)=\frac{P(q, \beta(q))}{R(q, \beta(q))} \in(-\infty, \infty)
$$

and $\beta^{*}\left(-\beta^{\prime}(q)\right)=-q \beta^{\prime}(q)+\beta(q)$.

Proof. Lemma 3.2(vii) ensures that there is one point where $\beta^{*}$ is greater than 0 . Now the concavity of $\beta^{*}$ gives this part.

Let $D=\operatorname{ess} \inf \inf _{i}\left(\left(\log p_{i}\right) /\left(\log r_{i}\right)\right)$. The result holds for the truncated process introduced in the proof of Lemma 3.2(v) (see [13, Proposition 3.3 II(b)] - the restriction there that $p_{i} \leq 1$ is not needed in the proof). Letting $c \rightarrow \infty$ gives $D_{L} \leq D$. For $\epsilon>0, r_{i}^{(D-\epsilon)}>p_{i}$. Then, for $q>0$,

$$
m(q,-q D)=\mathrm{E} \sum_{i} p_{i}^{q} r_{i}^{-q D}<\mathrm{E} \sum r_{i}^{-q D+q(D-\epsilon)}=\mathrm{E} \sum r_{i}^{-q \epsilon} \rightarrow 0
$$

as $q \rightarrow \infty$, so $\beta(q) \leq-q D$ for large positive $q$. Hence,

$$
\beta^{*}(D-\epsilon)=\inf _{q}\{(D-\epsilon) q+\beta(q)\} \leq-\epsilon q
$$

for large $q$, and this tends to $-\infty$. Consequently, $D_{L} \geq D$. The argument for $D_{U}$ is similar.

Once $\left(\Gamma_{L}-\epsilon, \Gamma_{U}+\epsilon\right) \subset\left(D_{L}, D_{U}\right),\left(D_{L}, D_{U}\right)$ is nonempty, and $\beta^{*}$ is continuous there, which forces $\beta^{*}\left(\Gamma_{U}\right)=\beta^{*}\left(\Gamma_{L}\right)=0$. By parts (vi) and (vii) of Lemma 3.2, $\tilde{q}\left(\Gamma_{U}\right) \leq 0$. By parts (vi) and (viii) of Lemma 3.2, $\tilde{q}\left(\Gamma_{L}\right) \geq 1$.

If $\beta$ is linear somewhere then, when it is, $1=m(q, \beta(q))=m(q, \gamma q+b)=\mathrm{E} \sum_{i} p_{i}^{q} r_{i}^{\gamma q+b}$, but this is possible only if $p_{i} r_{i}^{\gamma}=1$ for all $i$, which has been ruled out. The rest is calculus.

Theorem 6.1. Suppose that the open set condition and Assumption A hold, K has a $\gamma$-boundary, and $\left(D_{L}, D_{U}\right)$ is not empty. Suppose that, for some $\delta>0, m$ is finite in a neighbourhood of $(q, \beta(q))$ for every $q \in\left(\tilde{q}\left(\Gamma_{U}\right)-\delta, \tilde{q}\left(\Gamma_{L}\right)+\delta\right)$. Assume that, for some $\epsilon>0$,

$$
\begin{gathered}
\mathrm{E}\left[W^{\tilde{q}\left(\Gamma_{U}\right)-\epsilon}+W^{\tilde{q}\left(\Gamma_{L}\right)+\epsilon}\right]<\infty, \\
\mathrm{E}\left[\sum_{i} p_{i}^{\tilde{q}\left(\Gamma_{U}\right)-\epsilon}\right]<\infty,
\end{gathered}
$$

and

$$
\mathrm{E}\left[\left(\min _{i} r_{i}\right)^{-\tilde{q}\left(\Gamma_{L}\right) \Gamma_{L}-\gamma-\epsilon}\left(\sum_{i} p_{i}\right)^{\tilde{q}\left(\Gamma_{L}\right)+\epsilon}\right]<\infty .
$$

Then, P-a.s., $\operatorname{dim}_{H}\left(F_{\alpha}\right) \leq \beta^{*}(\alpha)$ if $\beta^{*}(\alpha) \geq 0$ and $F_{\alpha}=\varnothing$ if $\beta^{*}(\alpha)<0$. 
Proof. Using Lemma 6.1(iv), for suitably small $\varepsilon$ and $\varepsilon^{\prime}, \alpha \in\left(\Gamma_{L}+\varepsilon, \Gamma_{U}-\varepsilon\right)$ has $\tilde{q}(\alpha) \in$ $\left(\tilde{q}\left(\Gamma_{U}\right)-\varepsilon^{\prime}, \tilde{q}\left(\Gamma_{L}\right)+\varepsilon^{\prime}\right)$. Then (6.1) suffices to ensure that (5.2) holds for every $\tilde{q}(\alpha) \in$ $\left(\tilde{q}\left(\Gamma_{U}\right)-\varepsilon^{\prime}, \tilde{q}\left(\Gamma_{L}\right)+\varepsilon^{\prime}\right)$. Similarly, (6.2) suffices for (5.3) for every $\tilde{q}(\alpha) \in\left(\tilde{q}\left(\Gamma_{U}\right)-\varepsilon^{\prime}, 1\right]$ and (6.3) suffices for (5.4) for every $\tilde{q}(\alpha) \in\left(1, \tilde{q}\left(\Gamma_{L}\right)+\varepsilon^{\prime}\right)$.

For $\alpha>\alpha^{\circ}, \tilde{q}(\alpha)<0$ and, for $\alpha<\alpha^{\circ}, \tilde{q}(\alpha)>0$. Now as in the proof of Proposition 5.2, for $\alpha>\gamma>\alpha^{\circ}, F_{\alpha} \subset F_{\alpha} \subset F_{\gamma}$. Similarly, for $\alpha<\gamma<\alpha^{\circ}, F_{\alpha} \subset \overline{F_{\alpha}} \subset \overline{F_{\gamma}}$. We can use a single null set in obtaining $\operatorname{dim}_{H}\left(F_{\alpha}\right)$ for rational values of $\alpha$, and similarly for $\operatorname{dim}_{H}\left(\overline{F_{\alpha}}\right)$. Furthermore, $\beta^{*}$ is continuous and so the remaining values are forced by these.

\section{Partitioned self-similar measures}

The results in this section will be important for establishing the lower bound on $\operatorname{dim}_{H}\left(F_{\alpha}\right)$. They also yield a proof of Theorem 2.2, which relates to the basic 'fractal' property of $\mu_{\pi}$. Recall from Lemma 2.3 that $\mu_{\pi, i}(\cdot)=\mu_{i}\left(\left(\pi_{i}^{-1} \cdot\right) \cap C_{i}\right)$, that these are, given $\mathcal{B}_{1}$, independent copies of $\mu_{\pi}$, and that (2.6) holds for any $D \subset \mathbb{R}^{d}$. We assume throughout this section that parts (i) and (ii) of Assumption B hold, so the corresponding measures $v$ and $v_{\pi}$ can be constructed, and that the open set condition holds.

\subsection{Replacing int $K$ by $U$}

Later, we will need to bound the measure of small balls around a point $\pi(i) \in F$ and so we need such small balls to be sandwiched by sets whose measure we can easily estimate. Roughly, when $\pi(\boldsymbol{i}) \in \operatorname{int} K$, we can use $\operatorname{int}\left(K_{i \mid n}\right)$ for suitable $n$, but when $F$ is confined to the boundary of $K$, some additional manoeuvres are needed, drawing on, but modifying, ideas in [31]. These involve introducing new sets, $U_{i}$, with properties similar to the int $\left(K_{i}\right)$, but constructed so that $F \subset U$. When the strong open set condition holds, so that there is a positive probability that $F \cap \operatorname{int} K$ is nonempty, we will see that there is no need to introduce these sets.

For $\delta>0$, let the open set $K^{(\delta)}$ be given by $K^{(\delta)}=\bigcup_{x \in K} B(x ; \delta)$. Fix $\epsilon \in(0,1)$. For $i \in T$, let $G_{i}=S_{i}\left(K^{(2 \epsilon)}\right)$ and let

$$
\mathscr{H}(\boldsymbol{i})=\left\{\boldsymbol{j}: \sigma_{j} \leq \sigma_{\boldsymbol{i}}, G_{i} \cap K_{j} \neq \varnothing\right\}
$$

Note that $\mathscr{H}(\boldsymbol{i})$ contains $\boldsymbol{i}$ and all its ancestors and that if $\boldsymbol{k} \notin \mathscr{H}(\boldsymbol{i})$ then none of the descendants of $\boldsymbol{k}$ is in $\mathscr{H}(\boldsymbol{i})$ either. Now let

$$
\mathcal{I}(\boldsymbol{i})=\{\boldsymbol{j}: \boldsymbol{j} \in \mathscr{H}(\boldsymbol{i}) \text { but } \boldsymbol{j} \boldsymbol{k} \notin \mathscr{H}(\boldsymbol{i}) \text { for all } \boldsymbol{j} \boldsymbol{k} \in T\},
$$

so that $\mathcal{I}(\boldsymbol{i})$ contains those who are in $\mathscr{H}(\boldsymbol{i})$ but with no children in $\mathscr{H}(\boldsymbol{i})$-they are last in their line of descent to be in $\mathscr{H}(\boldsymbol{i})$. Note that $i \in \mathcal{I}(\boldsymbol{i})$. Let $M_{\boldsymbol{i}}$ be the cardinality of $\mathcal{I}(\boldsymbol{i})$. Under the open set condition, a consideration of volumes shows that there is a finite maximum number, $\hat{\mathfrak{n}}$, of nonoverlapping sets of shape int $K$ and diameter greater than 1 that can intersect with $K^{(2 \epsilon)}$. By arrangement, none of the members of $\mathcal{I}(\boldsymbol{i})$ is a descendant of any other and so the interiors of the sets indexed by $\mathcal{I}(\boldsymbol{i})$ do not overlap. Hence, rescaling by $\left(r_{i}\right)^{-1}$ shows that $M_{i} \leq \hat{\mathfrak{n}}$ for all $\boldsymbol{i} \in T$. (A different definition for $\mathcal{I}(\boldsymbol{i})$ is used in [31], with its members drawn from $\boldsymbol{j} \in \mathcal{C}\left(\sigma_{i}\right)$-this works there because the $r_{i}$ were assumed bounded away from 0 and so there is then a lower bound on the sizes of the sets indexed by $\mathcal{C}\left(\sigma_{i}\right)$ relative to that of $G_{i}$, allowing a volume argument to bound $M_{i}$.) Let $M$ be the largest integer such that $q=\mathrm{P}\left(M_{i}=M\right.$ for no $\left.i \in T\right)$ is strictly less than 1 , so $M$ is the essential supremum of the random variable $\sup _{i} M_{i}$. 
These definitions carry over to the tree emanating from $\boldsymbol{m}$. Let

$$
\mathscr{H}_{\boldsymbol{m}}(\boldsymbol{m i})=\left\{\boldsymbol{m j}: \sigma_{\boldsymbol{m} j} \leq \sigma_{\boldsymbol{m} i}, G_{\boldsymbol{m} i} \cap K_{\boldsymbol{m} j} \neq \varnothing\right\},
$$

which is the same as

$$
\left\{\boldsymbol{m} \boldsymbol{j}: \sigma_{\boldsymbol{m} j}-\sigma_{\boldsymbol{m}} \leq \sigma_{\boldsymbol{m} i}-\sigma_{\boldsymbol{m}}, S_{\boldsymbol{m}}^{-1} S_{\boldsymbol{m} \boldsymbol{i}}\left(K^{(2 \epsilon)}\right) \cap S_{\boldsymbol{m}}^{-1} S_{\boldsymbol{m} \boldsymbol{j}}(K) \neq \varnothing\right\},
$$

and so is indeed $\mathscr{H}$ on the tree with root $\boldsymbol{m}$. Then $\mathscr{H}_{\boldsymbol{m}}(\boldsymbol{m i}) \subset \mathscr{H}(\boldsymbol{m i})$ and if $\boldsymbol{m} \boldsymbol{j} \in \mathscr{H}(\boldsymbol{m i})$ then $\boldsymbol{m} \boldsymbol{j} \in \mathscr{H}_{\boldsymbol{m}}(\boldsymbol{m i})$. Now let

$$
\mathcal{I}_{\boldsymbol{m}}(\boldsymbol{m i})=\left\{\boldsymbol{j}: \boldsymbol{j} \in \mathscr{H}_{\boldsymbol{m}}(\boldsymbol{m i}) \text { but } \boldsymbol{j} \boldsymbol{k} \notin \mathscr{H}_{\boldsymbol{m}}(\boldsymbol{m i}) \text { for all } \boldsymbol{j} \boldsymbol{k} \in T\right\} .
$$

Then

$$
\begin{aligned}
& \tau_{\boldsymbol{m}}(\boldsymbol{m i})=\left\{\boldsymbol{j}: \boldsymbol{j} \in \mathscr{H}_{\boldsymbol{m}}(\boldsymbol{m i}) \text { but } \boldsymbol{j} \boldsymbol{k} \notin \mathscr{H}_{\boldsymbol{m}}(\boldsymbol{m i}) \text { for all } \boldsymbol{j} \boldsymbol{k} \in T\right\} \\
& =\left\{j: j \in \mathscr{H}_{\boldsymbol{m}}(\boldsymbol{m i}) \text { but } \boldsymbol{j} \boldsymbol{k} \notin \mathscr{H}(\boldsymbol{m i}) \text { for all } \boldsymbol{j} \boldsymbol{k} \in T\right\} \\
& \subset\{\boldsymbol{j}: \boldsymbol{j} \in \mathscr{H}(\boldsymbol{m i}) \text { but } \boldsymbol{j} \boldsymbol{k} \notin \mathscr{H}(\boldsymbol{m i}) \text { for all } \boldsymbol{j} \boldsymbol{k} \in T\} \\
& =\mathcal{I}(\boldsymbol{m i}) \text {, }
\end{aligned}
$$

and there must be equality here when $\left|\mathcal{I}_{\boldsymbol{m}}(\boldsymbol{m i})\right|=M$. Therefore, when any subtree from a first generation person contains a 'maximal' node, so does the original tree. Hence, with $q$ as defined earlier in this section, $q \leq \mathrm{E} q^{N}$, which forces $q=0$. Also, when $M_{i}=M$, the tree emanating from $i$ is independent of this information. Hence, the tree will contain nodes with $M_{i}=M$ in arbitrarily large generations. Order labels on a tree by generation and then lexicographically, so that the first node in the tree with a property can be sensibly identified.

Definition 7.1. Consider only the tree emanating from $i \in T$. Let $i k(i)$ be the first in this tree such that $\left|\mathcal{I}_{\boldsymbol{i}}(\boldsymbol{i} \boldsymbol{k}(\boldsymbol{i}))\right|=M$. Also, let $\boldsymbol{k}^{*}=\boldsymbol{k}(\varnothing)$.

Thus, $\boldsymbol{k}(\boldsymbol{i})$ is such that there are $M$ sets (with labels in $\mathcal{I}_{\boldsymbol{i}}(\boldsymbol{i} \boldsymbol{k}(\boldsymbol{i}))$ ) overlapping with $G_{\boldsymbol{i} \boldsymbol{k}(\boldsymbol{i})}$ in the tree emanating from $\boldsymbol{i}$. Since $\tau_{\boldsymbol{i}}(\boldsymbol{i} \boldsymbol{k}(\boldsymbol{i})) \subset \mathcal{I}(\boldsymbol{i} \boldsymbol{k}(\boldsymbol{i}))$ and the former has maximal cardinality, it must equal the latter. Therefore, all the $K_{j}$ overlapping $G_{i k(i)}$ with labels in $\mathcal{I}(\boldsymbol{i} \boldsymbol{k}(\boldsymbol{i}))$ must actually be in the tree emanating from $\boldsymbol{i}$. The next lemma uses these $\boldsymbol{k}(\boldsymbol{i})$ to construct the open set $U$ that will 'replace' int $K$. This has similar properties to int $K$, except that the self-similar measure puts some weight on $U$, whereas it could be concentrated on the boundary of $K$, and $U$ is random but int $K$ is not.

Lemma 7.1. Let

$$
U_{i}=\bigcup_{j} S_{i j k(i j)}\left(K^{(\epsilon)}\right) \quad \text { and } \quad U=U_{\varnothing} .
$$

Then $U_{i}$ is open, $U_{i} \subset U_{i-}, S_{i}^{-1}\left(U_{i}\right)$ has the same distribution as $U$, and $U_{i} \cap U_{i^{\prime}} \neq \varnothing$ only when $\boldsymbol{i} \leq \boldsymbol{i}^{\prime}$ or $\boldsymbol{i}^{\prime} \leq \boldsymbol{i}$. Furthermore,

$$
K_{i k(i)} \subset S_{i k(i)}\left(K^{(\epsilon)}\right) \subset U_{i}
$$

and $\mathrm{P}(F \cap U \neq \varnothing)=1$.

Proof. The first three assertions are immediate. Suppose that $y \in U_{\boldsymbol{i}} \cap U_{\boldsymbol{i}^{\prime}}$, so there are $\boldsymbol{i} \boldsymbol{j}$ and $\boldsymbol{i}^{\prime} \boldsymbol{j}^{\prime}$ such that

$$
y \in S_{i j k(i j)}\left(K^{(\epsilon)}\right) \cap S_{i^{\prime} j^{\prime} \boldsymbol{k}\left(\boldsymbol{i}^{\prime} \boldsymbol{j}^{\prime}\right)}\left(K^{(\epsilon)}\right) .
$$


Let $\boldsymbol{l}=\boldsymbol{i} \boldsymbol{j} \boldsymbol{k}(\boldsymbol{i} \boldsymbol{j})$ and $\boldsymbol{l}^{\prime}=\boldsymbol{i}^{\prime} \boldsymbol{j}^{\prime} \boldsymbol{k}\left(\boldsymbol{i}^{\prime} \boldsymbol{j}^{\prime}\right)$. Say $\sigma_{\boldsymbol{l}} \leq \sigma_{\boldsymbol{l}^{\prime}}$, or, equivalently, $r_{\boldsymbol{l}} \geq r_{\boldsymbol{l}^{\prime}}$. The idea is to show that $\boldsymbol{i} \boldsymbol{j} \leq \boldsymbol{l}^{\prime}=\boldsymbol{i}^{\prime} \boldsymbol{j}^{\prime} \boldsymbol{k}\left(\boldsymbol{i}^{\prime} \boldsymbol{j}^{\prime}\right)$, which implies that either $\boldsymbol{i} \leq \boldsymbol{i}^{\prime}$ or $\boldsymbol{i}^{\prime} \leq \boldsymbol{i}$.

There is $y_{1} \in S_{l}(K)$ with $\left|y_{1}-y\right|<r_{l} \epsilon$ and $y_{2} \in S_{l^{\prime}}(K)=K_{l^{\prime}}$ with $\left|y_{2}-y\right|<r_{l^{\prime}} \epsilon$. Hence, $\left|y_{1}-y_{2}\right|<2 r_{l} \epsilon$. Therefore,

$$
y_{2} \in\left(S_{l}(K)\right)^{\left(2 r_{l} \epsilon\right)} \cap K_{l^{\prime}}=S_{l}\left(K^{(2 \epsilon)}\right) \cap K_{l^{\prime}}=G_{l} \cap K_{l^{\prime}},
$$

and so $G_{\boldsymbol{l}} \cap K_{\boldsymbol{l}^{\prime}}$ is nonempty. Then, since $\sigma_{\boldsymbol{l}} \leq \sigma_{\boldsymbol{l}^{\prime}}$, some ancestor of $\boldsymbol{l}^{\prime}$ is in $\mathcal{I}(\boldsymbol{l})$ and, therefore (from the definition of $\boldsymbol{k}(\cdot)$ ), must be in $\mathcal{I}_{\boldsymbol{i}}(\boldsymbol{l})$ and, hence, in the tree emanating from $\boldsymbol{i} \boldsymbol{j}$. Thus, $\boldsymbol{i} \boldsymbol{j} \leq \boldsymbol{l}^{\prime}=\boldsymbol{i}^{\prime} \boldsymbol{j}^{\prime} \boldsymbol{k}\left(\boldsymbol{i}^{\prime} \boldsymbol{j}^{\prime}\right)$, giving the result.

The final assertions follow directly from the definitions and noting that $F \cap K_{k}(\varnothing) \neq \varnothing$.

\subsection{Estimates of the measure}

When we come to estimate the measures of open balls, we need an upper bound on the measure of the open set that contains them, which may be $U$ or int $K$ depending on the formulation. The next two lemmas provide these. Recall that $\mu_{\pi}$ is a particular case of $\nu_{\pi}$, so the second part in the first lemma is just for emphasis and Lemma 2.6 is a special case of the second one.

Lemma 7.2. We have $v_{\pi}\left(U_{i}\right) \leq y_{i} Y_{i}$. In particular, $\mu_{\pi}\left(U_{i}\right) \leq p_{i} W_{i}$.

Proof. Let $\mathcal{K}(\boldsymbol{i} ; s)=\left\{\boldsymbol{j}: \sigma_{\boldsymbol{i} \boldsymbol{j} \boldsymbol{k}(\boldsymbol{i} \boldsymbol{j})} \leq s\right\}$. Now truncate $U_{\boldsymbol{i}}$ by letting

$$
U_{i}(s)=\bigcup_{j \in \mathcal{K}(i ; s)} S_{i j k(i j)}\left(K^{(\epsilon)}\right) .
$$

Then, for any $n$, as in (2.7),

$$
v_{\pi}\left(U_{i}(s)\right) \leq \sum_{j \in T_{n}} y_{j} Y_{j} I\left(U_{i}(s) \cap K_{j} \neq \varnothing\right) .
$$

By Lemma 2.1, there is an $n^{\prime} \geq|\boldsymbol{i}|$ such that $\inf \left\{\sigma_{k}: \boldsymbol{k} \in T_{n}\right\}>s$ for all $n \geq n^{\prime}$. Take $n \geq n^{\prime}$. Suppose that $y \in U_{\boldsymbol{i}}(s) \cap K_{\boldsymbol{j}}$. Then there is $\boldsymbol{l}^{\prime}=\boldsymbol{i}_{\boldsymbol{j}} \boldsymbol{k}\left(\boldsymbol{i} \boldsymbol{j}^{\prime}\right)$, necessarily with $\sigma_{\boldsymbol{l}^{\prime}} \leq s<\sigma_{\boldsymbol{j}}$, and with

$$
y \in S_{l^{\prime}}\left(K^{(\epsilon)}\right) \cap K_{j}
$$

Since $S_{\boldsymbol{l}^{\prime}}\left(K^{(\epsilon)}\right) \subset G_{\boldsymbol{l}^{\prime}}$, this implies that $G_{\boldsymbol{l}^{\prime}} \cap K_{\boldsymbol{j}} \neq \varnothing$. Since $\sigma_{\boldsymbol{l}^{\prime}} \leq \sigma_{\boldsymbol{j}}$, some ancestor of $\boldsymbol{j}$ is in $\mathcal{I}\left(\boldsymbol{l}^{\prime}\right)$, and, therefore (from the definition of $\boldsymbol{k}(\cdot)$ ), must be in the tree emanating from $\boldsymbol{i} \boldsymbol{j}^{\prime}$, and, hence, from $i$. Hence, for all $n \geq n^{\prime}$,

$$
\begin{aligned}
\sum_{\boldsymbol{j} \in T_{n}} y_{\boldsymbol{j}} Y_{\boldsymbol{j}} I\left(U_{\boldsymbol{i}}(s) \cap K_{\boldsymbol{j}} \neq \varnothing\right) & =\sum_{\left\{\boldsymbol{j}: \boldsymbol{i} \boldsymbol{j} \in T_{n}\right\}} y_{\boldsymbol{i} \boldsymbol{j}} Y_{\boldsymbol{i} \boldsymbol{j}} I\left(U_{\boldsymbol{i}}(s) \cap K_{\boldsymbol{i} \boldsymbol{j}} \neq \varnothing\right) \\
& \leq \sum_{\left\{\boldsymbol{j}: \boldsymbol{i} \boldsymbol{j} \in T_{n}\right\}} y_{\boldsymbol{i} \boldsymbol{j}} Y_{i \boldsymbol{j}} \\
& =y_{\boldsymbol{i}} Y_{\boldsymbol{i}},
\end{aligned}
$$

using (3.3). This shows that $v_{\pi}\left(U_{i}(s)\right) \leq y_{i} Y_{i}$ for any $s$, and upon letting $s \uparrow \infty$, monotone convergence gives $v_{\pi}\left(U_{i}\right) \leq y_{i} Y_{i}$.

Lemma 7.3. We have $v_{\pi}\left(\operatorname{int}\left(K_{i}\right)\right) \leq y_{i} Y_{i}$.

Proof. The proof is the same as Lemma 2.6, but with $y_{j} Y_{j}$ in place of $p_{j} W_{j}$. 


\subsection{Self-similar measures partition}

Let $\mathscr{D}(\cdot, \cdot)$ give the shortest distance between its two arguments. Take $V=U$. If $\mathrm{P}(F \cap$ $\operatorname{int} K=\varnothing)<1$, we can instead take $V=\operatorname{int} K$. Most arguments are unchanged; those that are not are indicated.

Recall, from Section 2, that, for $i \in \partial T, K_{i}$ is another notation for $\pi(i)$ and that $V^{\mathrm{c}}$ is the complement of $V$. For $i \in T \cup \partial T$, let

$$
\Delta_{i}=-\log \left(\mathscr{D}\left(K_{i}, V^{\mathrm{c}}\right)\right)
$$

and, for $\boldsymbol{i} \in T$ and $\boldsymbol{j} \in T \cup \partial T$, let

$$
\Delta_{i j}^{i}=-\log \left(\frac{\mathscr{D}\left(K_{i j}, V_{i}^{\mathrm{c}}\right)}{r_{i}}\right)=-\log \mathscr{D}\left(K_{i j}, V_{i}^{\mathrm{c}}\right)-\sigma_{i},
$$

which, given the information up to generation $|\boldsymbol{i}|$, has the same distribution as $\Delta_{j}$. The basic idea is to show that $\Delta: \partial T \rightarrow \mathbb{R}$ is usually not large, where 'usually' is with respect to the measure $v$, or $\mu$, on $\partial T$. It is worth noting that the random variable $\Delta_{i}$ depends, through $U$, on a random portion of the tree, and not just on the tree up to generation $|\boldsymbol{i}|$. This difficulty is absent when $V=\operatorname{int} K$.

Lemma 7.4. For any $i \in T, j \in T \cup \partial T$, and $c$,

$$
I\left(\Delta_{i j}>\sigma_{i j}+c\right) \leq I\left(\Delta_{i}>\sigma_{i}+c\right) I\left(\Delta_{i j}^{i}>\sigma_{i j}-\sigma_{i}+c\right) .
$$

Proof. Since $K_{i j} \subset K_{i}, \Delta_{i j} \leq \Delta_{i}$ and, since $V_{i} \subset V$,

$$
\Delta_{i j}=-\log \left(\mathscr{D}\left(S_{i j} K, V^{\mathrm{c}}\right)\right) \leq-\log \left(\mathscr{D}\left(S_{i j} K, V_{i}^{\mathrm{c}}\right)\right)=\Delta_{i j}^{i}+\sigma_{i}
$$

Hence,

$$
I\left(\Delta_{i j}>\sigma_{i j}+c\right) \leq I\left(\Delta_{i}>\sigma_{i j}+c\right) I\left(\Delta_{i j}^{i}>\sigma_{i j}-\sigma_{i}+c\right)
$$

and $\sigma_{i j} \geq \sigma_{i}$.

Now certain indicator variables are introduced. These are defined differently depending on whether $V$ is $U$ or int $K$, but have the same relevant properties. Both depend on an integer $r$ and a suitable $\epsilon \in(0,1)$.

When $V=U$, the definition of $U$ (or, more precisely, $G_{i}$ ) supplies the $\epsilon$. Let

$$
\kappa(\boldsymbol{k})=\sup \left\{|\boldsymbol{i}|: \boldsymbol{i} \in \mathcal{C}\left(\sigma_{\boldsymbol{k}}\right)\right\}=\sup \left\{|\boldsymbol{i}|: \sigma_{\boldsymbol{i}} \leq \sigma_{\boldsymbol{k}}<\sigma_{\boldsymbol{i}}\right\},
$$

which is finite, by Lemma 2.1 , and at least $|\boldsymbol{k}|+1$. Recall that $\boldsymbol{k}^{*}=\boldsymbol{k}(\varnothing)$ was introduced in Definition 7.1. Let $r$ be sufficiently large that, with positive probability, $\kappa\left(\boldsymbol{k}^{*}\right) \leq r$, and, for $i \in T_{r}$, let

$$
\xi_{i}=1-I\left(\kappa\left(\boldsymbol{k}^{*}\right) \leq r, \boldsymbol{k}^{*} \leq \boldsymbol{i}\right),
$$

so there is a positive probability that at least one of these is 0 . For $\boldsymbol{k}$ with $|\boldsymbol{k}| \leq r-1$, $I(\kappa(\boldsymbol{k}) \leq r)$ is known given $\mathcal{B}_{r}$. Then $I\left(M_{\boldsymbol{k}}=M\right)$ is also known, since all those born before $\sigma_{k}$ are in generations up to $r-1$. Hence, $\xi_{i}$ is not random given $\mathscr{B}_{r}$. (Note that this need not be so if ' $\kappa\left(\boldsymbol{k}^{*}\right) \leq r$ ' is omitted.)

When $\mathrm{P}(F \cap \operatorname{int} K \neq \varnothing)>0$ (and $V=\operatorname{int} K)$, choose $r$ large enough that

$$
\mathrm{P}\left(K_{i} \subset \operatorname{int} K \neq \varnothing \text { for some } i \in T_{r}\right)>0 .
$$


Now choose $\epsilon \in(0,1)$ and small enough that there is a positive probability that at least one of the variables defined for $i \in T_{r}$ by $\xi_{i}=I\left(\mathscr{D}\left(K_{i},(\operatorname{int} K)^{\mathrm{c}}\right)<r_{i} \epsilon\right)$ is 0 . Note that $\xi_{i}=I\left(\Delta_{i}>\sigma_{i}-\log \epsilon\right)$.

In both cases we may now define $c^{*}=-\log \epsilon \in(0, \infty)$ and $\gamma$ by

$$
\mathrm{E}\left[\sum_{\boldsymbol{i} \in T_{r}} y_{\boldsymbol{i}} \xi_{\boldsymbol{i}}\right]=\gamma^{r}
$$

Then $\gamma<1$ because $\mathrm{E}\left[\sum_{i \in T_{r}} y_{i}\right]=1$.

Lemma 7.5. For all $n \in \mathbb{N}$,

$$
\mathrm{E}\left[\sum_{j \in T_{n}} y_{j} I\left(\Delta_{j}>\sigma_{j}+c^{*}\right)\right] \leq \gamma^{n-r} .
$$

Proof. Let

$$
\chi_{i}=I\left(\Delta_{i}>\sigma_{i}+c^{*}\right) \quad \text { and } \quad \chi_{i j}^{i}=I\left(\Delta_{i j}^{i}>\sigma_{i j}-\sigma_{i}+c^{*}\right) .
$$

Then, by Lemma $7.4, \chi_{i j} \leq \chi_{i} \chi_{i j}^{i}$ so that

$$
\sum_{i j \in T_{n+r}} y_{i j} \chi_{i j} \leq \sum_{i \in T_{r}} y_{i} \chi_{i}\left(\sum_{\left\{j: i j \in T_{n+r}\right\}} \frac{y_{i j}}{y_{i}} \chi_{i j}^{i}\right) .
$$

Note that $\left\{y_{i} \chi_{i}:|\boldsymbol{i}|=r\right\}$ are not necessarily known when given $\mathcal{B}_{r}$. However, as we shall see, $\chi_{i} \leq \xi_{i}$. Hence,

$$
\sum_{\boldsymbol{i} \in T_{n+r}} y_{i j} \chi_{i j} \leq \sum_{i \in T_{r}} y_{i} \xi_{i}\left(\sum_{\left\{\boldsymbol{j}: \boldsymbol{i j} \in T_{n+r}\right\}} \frac{y_{i j}}{y_{i}} \chi_{i j}^{i}\right) .
$$

Now, given $\mathcal{B}_{r}$, each term in the brackets on the right-hand side is an independent copy of $\sum_{i \in T_{n}} y_{i} \chi_{i}$ and $\left\{y_{i} \xi_{i}: i \in T_{r}\right\}$ are known. Thus, taking expectations conditional on $\mathscr{B}_{r}$ and then unconditionally,

$$
\mathrm{E}\left[\sum_{i j \in T_{n+r}} y_{i j} \chi_{i j}\right] \leq \mathrm{E}\left[\sum_{i \in T_{r}} y_{i} \xi_{i}\right] \mathrm{E}\left[\sum_{i \in T_{n}} y_{i} \chi_{i}\right]=\gamma^{r} \mathrm{E}\left[\sum_{i \in T_{n}} y_{i} \chi_{i}\right] .
$$

The proof is finished by induction. It remains to show that, when $\xi_{i}$ is 0 , so is $\chi_{i}$. When $V=\operatorname{int} K$, by definition, $\xi_{i}$ is the same as $\chi_{i}$ and there is nothing more to do. Turning to the other case, when $V=U$, take $i$ with $\xi_{i}=0$ so that, by definition, $\boldsymbol{k}^{*} \leq \boldsymbol{i}$. Then, by Lemma 7.4, $\chi_{\boldsymbol{i}} \leq \chi_{\boldsymbol{k}}^{*}$ for any $\boldsymbol{i} \geq \boldsymbol{k}^{*}$. Since $K_{\boldsymbol{k}^{*}} \subset S_{\boldsymbol{k}^{*}}\left(K^{(\epsilon)}\right) \subset V$,

$$
\Delta_{\boldsymbol{k}^{*}}=-\log \mathcal{D}\left(K_{\boldsymbol{k}^{*}}, V^{\mathrm{c}}\right) \leq-\log \mathcal{D}\left(K_{\boldsymbol{k}^{*}},\left(S_{\boldsymbol{k}^{*}}\left(K^{(\epsilon)}\right)\right)^{\mathrm{c}}\right) \leq-\log \left(r_{\boldsymbol{k}^{*}} \epsilon\right)=\sigma_{\boldsymbol{k}^{*}}+c^{*},
$$

which means that $\chi_{\boldsymbol{k}^{*}}=0$. Hence, $\chi_{\boldsymbol{i}}=0$ as required.

Proposition 7.1. We have $\mathrm{E} v_{\pi}\left(V^{\mathrm{c}}\right)=0$ and $\mathrm{E} v_{\pi}\left(\left(\bigcup_{i \in T_{1}} V_{i}\right)^{\mathrm{c}}\right)=0$. Let

$$
F_{*}=\bigcap_{n}\left(\bigcup_{j \in T_{n}} V_{j}\right) \text {. }
$$

Then $\mathrm{E} v_{\pi}\left(F_{*}^{\mathrm{c}}\right)=0$ and so $\mathrm{E} v_{\pi}\left(\left(F \cap F_{*}\right)^{\mathrm{c}}\right)=0$. In particular, all these results hold for $\mu_{\pi}$. 
Proof. Note that $\pi(\boldsymbol{j}) \in V^{\mathrm{c}}$ implies that $\Delta_{\boldsymbol{j} \mid n}=\infty$ for every $n$. Hence,

$$
\mathrm{E} v_{\pi}\left(V^{\mathrm{c}}\right) \leq \mathrm{E} \sum_{i \in T_{n}} y_{i} Y_{i} I\left(\Delta_{i}=\infty\right) \leq \mathrm{E}\left[\sum_{j \in T_{n}} y_{j} I\left(\Delta_{j}>\sigma_{j}+c^{*}\right)\right] \leq \gamma^{n-r} \rightarrow 0
$$

using Lemma 7.5. Let $v_{i}$ be the copy of $v$ defined on the tree emanating from $\boldsymbol{i}$. Then

$$
\nu(A)=\sum_{i \in T_{n}} y_{i} v_{i}\left(A \cap C_{i}\right) .
$$

Hence,

$$
\begin{aligned}
\nu\left(\left\{\boldsymbol{i}: \pi(\boldsymbol{i}) \in\left(\bigcup_{\boldsymbol{j} \in T_{n}} V_{\boldsymbol{j}}\right)^{\mathrm{c}}\right\}\right) & =\sum_{\boldsymbol{k} \in T_{n}} y_{\boldsymbol{k}} \nu_{\boldsymbol{k}}\left(\left\{\boldsymbol{i}: \pi(\boldsymbol{i}) \in\left(\bigcup_{\boldsymbol{j} \in T_{n}} V_{\boldsymbol{j}}\right)^{\mathrm{c}}\right\} \cap C_{\boldsymbol{k}}\right) \\
& \leq \sum_{\boldsymbol{k} \in T_{n}} y_{\boldsymbol{k}} \nu_{\boldsymbol{k}}\left(\left\{\boldsymbol{i}: \pi(\boldsymbol{i}) \in V_{\boldsymbol{k}}^{\mathrm{c}}\right\} \cap C_{\boldsymbol{k}}\right),
\end{aligned}
$$

and, given $\mathcal{B}_{n}$, each term on the right-hand side has expectation 0 . Hence, taking $n=1$ gives $\mathrm{E} v_{\pi}\left(\left(\cup_{i \in T_{1}} V_{i}\right)^{\mathrm{c}}\right)=0$ and, more generally,

$$
\mathrm{E} v_{\pi}\left(\left\{\pi(\boldsymbol{i}) \in \bigcup_{n}\left(\bigcup_{\boldsymbol{j} \in T_{n}} V_{\boldsymbol{j}}\right)^{\mathrm{c}}\right\}\right)=0,
$$

which implies that $\mathrm{E} v_{\pi}\left(F_{*}^{\mathrm{c}}\right)=0$. We already know that $v_{\pi}\left(F^{\mathrm{c}}\right)=0$, so $\mathrm{E} v_{\pi}\left(\left(F \cap F_{*}\right)^{\mathrm{c}}\right)=0$.

Proof of Theorem 2.2. The $\left\{V_{i}\right\}$ introduced above $\left(\left\{U_{i}\right\}\right.$, or $\left\{\operatorname{int} K_{i}\right\}$ when $\mathrm{P}(F \cap \operatorname{int} K \neq$ $\varnothing)>0)$ are disjoint and, by Proposition 7.1, E $\mu_{\pi}\left(\left(\bigcup_{i \in T_{1}} V_{i}\right)^{\mathrm{c}}\right)=0$. Also, for $i \neq j$, $S_{i}^{-1} V_{i}$ and $S_{i}^{-1} V_{j}$ are disjoint and so $S_{i}^{-1} V_{j} \subset\left(S_{i}^{-1} V_{i}\right)^{\mathrm{c}}$. Applying Proposition 7.1 to $\mu_{\pi, i}$, $\mathrm{E} \mu_{\pi, i}\left(\left(S_{i}^{-1} V_{i}\right)^{\mathrm{c}}\right)=0$. Then, using decomposition (2.6), excluding a suitable P-null set,

$$
\begin{aligned}
\mu_{\pi}(D) & =\mu_{\pi}\left(D \cap\left(\bigcup_{j \in T_{1}} V_{j}\right)\right) \\
& =\sum_{i} p_{i} \mu_{\pi, i}\left(S_{i}^{-1}\left(D \cap\left(\bigcup_{j \in T_{1}} V_{j}\right)\right)\right) \\
& =\sum_{i} p_{i} \mu_{\pi, i}\left(S_{i}^{-1} D \cap\left(\bigcup_{j \in T_{1}} S_{i}^{-1} V_{j}\right)\right) \\
& =\sum_{i} p_{i} \mu_{\pi, i}\left(S_{i}^{-1} D \cap S_{i}^{-1} V_{i}\right) \\
& =\sum_{i} p_{i} \mu_{\pi, i}\left(S_{i}^{-1}\left(D \cap V_{i}\right)\right),
\end{aligned}
$$

as required.

\section{Lower bound on $\operatorname{dim}_{H}\left(F_{\alpha}\right)$}

\subsection{The result}

The aim is to give conditions for the upper bound derived in Proposition 5.1 to be also a lower bound. When Assumption B holds, let $\alpha$ be given by $\alpha=P(q, \beta) / R(q, \beta)$, where $P$ and $R$ 
are as in (3.1) and (3.2). Then the idea is to show that, under suitable weak moment conditions, $\operatorname{dim}_{H}\left(F_{\alpha}\right) \geq q \alpha+\beta$. The next lemma shows that this lower bound is indeed positive.

Lemma 8.1. When Assumption B holds, $\beta^{*}(\alpha)=q \alpha+\beta>0$ and in Lemma 3.2(vi) $\tilde{q}(\alpha)=q$ and $\beta(\tilde{q}(\alpha))=\beta$.

Proof. Note first that

$$
0<\mathrm{E}\left[-\sum y_{i} \log y_{i}\right]=-\mathrm{E} \sum_{i} p_{i}^{q} r_{i}^{\beta}\left(q \log p_{i}+\beta \log r_{i}\right)=q P+\beta R .
$$

Hence, $(q \alpha+\beta) R>0$, and so, since $R \in(0, \infty), q \alpha+\beta>0$. Lemma 3.2(viii) combines with Assumption $\mathrm{B}(\mathrm{i})$ to give $\beta^{*}(\alpha)=q \alpha+\beta, \tilde{q}(\alpha)=q$, and $\beta(\tilde{q}(\alpha))=\beta$.

When Assumption B holds, the measure $v$ has already been defined on $\partial T$ through $v\left(C_{\boldsymbol{i}}\right)=$ $y_{i} Y_{i}$ and $v_{\pi}$ is its projection onto $\mathbb{R}^{d}$.

Proposition 8.1. Suppose that Assumptions $A$ and $B$ and the open set condition hold. Suppose also that $\mathrm{E} Y \log _{+} Y<\infty$ and that $\mathrm{E} Y|\log W|<\infty$. Then $\operatorname{dim}_{H}\left(F_{\alpha}\right) \geq \beta^{*}(\alpha)$ a.s.

This result supplies the second half of Theorem 3.1. We have not obtained a more refined result of the null set here remaining the same as $\alpha$ varies. This is done for measures on $\partial T$ in [3] under additional conditions. We tackle the lower bound using the following lemma, with $\nu_{\pi}$ as $\mathfrak{m}$ and the set $A$ given by $\pi\left(G^{*}\right)$, the projection of a suitable $G^{*} \subset \partial T$ into $\mathbb{R}^{d}$. Then we give a number of lemmas to break the main proof down into digestible chunks. The first few of these show that various relevant sets in $\partial T$ have full measure under $v$.

Lemma 8.2. (Theorem 4.9 of [12].) Let $\mathfrak{m}$ be a Borel measure on $\mathbb{R}^{d}$ and $A$ an $\mathfrak{m}$-measurable set with $\mathfrak{m}(A)>0$. If, for all $x \in A$,

$$
\underset{r \downarrow 0}{\limsup } \frac{\mathfrak{m}(B(x ; r))}{r^{s}}<\infty
$$

then $\operatorname{dim}_{H}(A) \geq s$.

Lemma 8.3. Let

$$
G_{1}=\left\{i \in \partial T: \lim _{n \rightarrow \infty} \frac{\log Y_{i \mid n}}{\log r_{i \mid n}}=0\right\} .
$$

If $\mathrm{E}\left[Y \log _{+} Y\right]<\infty$ then $v\left(G_{1}\right)=v(\partial T)$, P-a.s.

Proof. Let

$$
E_{\epsilon, k}=\left\{i \in \partial T: \frac{\left|\log Y_{i \mid k}\right|}{-\log r_{i \mid k}}>\epsilon\right\}
$$

Then, for any $l \in \mathbb{N}$,

$$
\left\{i \in \partial T: \limsup _{n \rightarrow \infty} \frac{\left|\log Y_{i \mid n}\right|}{-\log r_{i \mid n}}>\epsilon\right\} \subset \bigcup_{k \geq l} E_{\epsilon, k} .
$$

Hence, for any $\epsilon>0$,

$$
v\left\{i \in \partial T: \limsup _{n \rightarrow \infty} \frac{\left|\log Y_{i \mid n}\right|}{-\log r_{i \mid n}}>\epsilon\right\} \leq \lim _{l \uparrow \infty} \sum_{k \geq l} v\left(E_{\epsilon, k}\right) .
$$


Now

$$
\begin{aligned}
\mathrm{E}\left[v\left(E_{\epsilon, n}\right)\right] & =\mathrm{E}\left[\sum_{i \in T_{n}} y_{i} Y_{i} I\left(\left|\log Y_{i}\right|>-\epsilon \log r_{i}\right)\right] \\
& =\mathrm{E}\left[\sum_{i \in T_{n}} p_{i}^{q} r_{i}^{\beta} \mathrm{E}\left[Y_{i} I\left(\left|\log Y_{i}\right|>-\epsilon \log r_{i}\right) \mid \mathcal{B}_{n}\right]\right] \\
& =\widetilde{\mathrm{E}}\left[\mathrm{E} Y I\left(|\log Y|>\epsilon S_{n}\right)\right],
\end{aligned}
$$

using Lemma 4.1. Recall that $U(t)$ is the renewal function for $S_{n}$, for which there is a constant $C$ such that $U(t) \leq C(t+1)$. Summing over $n$,

$$
\begin{aligned}
\sum_{n} \mathrm{E}\left[v\left(E_{\epsilon, n}\right)\right] & =\sum_{n} \widetilde{\mathrm{E}}\left[\mathrm{E} Y I\left(|\log Y|>\epsilon S_{n}\right)\right] \\
& =\mathrm{E}\left[Y U\left(\epsilon^{-1}|\log Y|\right)\right] \\
& \leq C \mathrm{E}\left[Y\left(1+\epsilon^{-1}|\log Y|\right)\right]
\end{aligned}
$$

which is finite when $\mathrm{E}\left[Y \log _{+} Y\right]<\infty$. Hence, the right-hand side of (8.1) is 0 a.s.

Lemma 8.4. Let

$$
G_{2}=\left\{i \in \partial T: \lim _{n \rightarrow \infty} \frac{\log W_{i \mid n}}{\log r_{i \mid n}}=0\right\} .
$$

If $\mathrm{E} Y|\log W|<\infty$ then $v\left(G_{2}\right)=v(\partial T)$, P-a.s.

Proof. The proof is the same as the previous proof, but with $\log W$ in the place of $\log Y$.

In the same way, the following minor variant can be established. Using it in place of Lemma 8.4, it is possible to give lower bounds for $\operatorname{dim}_{H}\left(\underline{F_{\alpha}}\right)$ when $q<0$ and for $\operatorname{dim}_{H}\left(\overline{F_{\alpha}}\right)$ when $q>0$.

Lemma 8.5. If $\mathrm{E} Y \log _{+} W<\infty$,

$$
\mathrm{Ev}\left\{i \in \partial T: \liminf _{n \rightarrow \infty} \frac{\log W_{i \mid n}}{\log r_{i \mid n}}<0\right\}=0 .
$$

If $\mathrm{E} Y \log _{+}(1 / W)<\infty$,

$$
\mathrm{E} v\left\{i \in \partial T: \limsup _{n \rightarrow \infty} \frac{\log W_{i \mid n}}{\log r_{\boldsymbol{i} \mid n}}>0\right\}=0 .
$$

Lemma 8.6. Let

$$
G_{3}=\left\{i \in \partial T: \lim _{n \rightarrow \infty} \frac{\log r_{i \mid n}}{n}=\mathrm{E}\left[\sum_{i} p_{i}^{q} r_{i}^{\beta} \log r_{i}\right]\right\}
$$

and

$$
G_{4}=\left\{i \in \partial T: \lim _{n \rightarrow \infty} \frac{\log p_{i \mid n}}{n}=\mathrm{E}\left[\sum_{i} p_{i}^{q} r_{i}^{\beta} \log p_{i}\right]\right\} .
$$

Then $v\left(G_{3}\right)=v\left(G_{4}\right)=v(\partial T)$, P-a.s. On $G_{3} \cap G_{4}, \log p_{i \mid n} / \log r_{i \mid n} \rightarrow \alpha$ as $n \rightarrow \infty$. 
Proof. This is a standard, using the strong law of large numbers for what is sometimes called the Peyrière measure: see, for example, the proofs of Lemma 11 of [25], Lemma 4.6 of [2], and Theorem 8 of [4]. It uses Assumption B(iii) and (iv).

The final result needed of this kind, which is stated next, concerns the variables $\Delta_{j i}^{j}$ introduced in (7.2). It seems possible that it can be proved by a suitable application of the ergodic theorem to the Peyrière measure, but we have not tried to do this. Before proving it a couple of additional lemmas are needed that concern $\Delta_{i}\left(=\Delta_{\varnothing i}^{\varnothing}\right)$ defined in (7.1). In the proofs $c^{*}$ and $\gamma$ are as defined just before Lemma 7.5.

Lemma 8.7. Let

$$
G_{5}=\left\{i \in \partial T: \frac{\Delta_{i}^{i \mid k}}{k} \rightarrow 0\right\}
$$

Then $v\left(G_{5}\right)=v(\partial T)$, P-a.s.

Lemma 8.8. For large $C^{\prime}>0$,

$$
\sum_{k} \operatorname{Ev}\left(\left\{i \in \partial T: \Delta_{i}>k C^{\prime}\right\}\right)<\infty .
$$

Proof. First, with $C^{\prime}=C+c^{*}$

$$
\begin{aligned}
\left\{\Delta_{i}>k C^{\prime}\right\} & \subset\left\{\Delta_{i}>\sigma_{i \mid k}+c^{*}\right\} \cup\left\{\sigma_{\boldsymbol{i} \mid k}+c^{*}>k\left(C+c^{*}\right)\right\} \\
& \subset\left\{\Delta_{\boldsymbol{i}}>\sigma_{\boldsymbol{i} \mid k}+c^{*}\right\} \cup\left\{\sigma_{\boldsymbol{i} \mid k}>k C\right\} .
\end{aligned}
$$

Then

$$
\sum_{k} \mathrm{E} v\left(\left\{\boldsymbol{i} \in \partial T: \sigma_{i \mid k}>k C\right\}\right)=\sum_{k} \mathrm{E} \sum_{j \in T_{k}} y_{j} Y_{j} I\left(\sigma_{j}>k C\right)=\sum_{k} \widetilde{\mathrm{P}}\left(S_{k}>k C\right),
$$

which is finite when $C>\widetilde{\mathrm{E}} S_{1}$ and $\widetilde{\mathrm{E}} S_{1}^{2}<\infty$, using a result of Hsu and Robbins [11, Corollary 2, Section 10.4]. Assumption B(iv) implies that $\widetilde{\mathrm{E}} S_{1}^{2}<\infty$. Also, since $\Delta_{i} \leq \Delta_{i \mid k}$, we have

$$
\begin{aligned}
\sum_{k} \operatorname{Ev}\left(\left\{\boldsymbol{i} \in \partial T: \Delta_{\boldsymbol{i}}>\sigma_{\boldsymbol{i} \mid k}+c^{*}\right\}\right) & \leq \sum_{k} \operatorname{Ev}\left(\left\{\boldsymbol{i} \in \partial T: \Delta_{\boldsymbol{i} \mid k}>\sigma_{\boldsymbol{i} \mid k}+c^{*}\right\}\right) \\
& =\sum_{k}\left[\sum_{\boldsymbol{j} \in T_{k}} y_{\boldsymbol{j}} Y_{\boldsymbol{j}} I\left(\Delta_{\boldsymbol{j}}>\sigma_{\boldsymbol{j}}+c^{*}\right)\right] \\
& \leq \sum_{k} \gamma^{k-r}
\end{aligned}
$$

by Lemma 7.5. Combining these two bounds gives the result.

Lemma 8.9. If $\mathrm{E} \sum_{k} v\left(\left\{\boldsymbol{i} \in \partial T: \Delta_{i}>k C\right\}\right)$ is finite for some $C>0$ then it is finite for all $C \in(0, \infty)$.

Proof. For any $C>0$,

$$
\int_{\partial T} \Delta_{i} v(\mathrm{~d} \boldsymbol{i}) \leq C \sum_{k} v\left(\left\{\boldsymbol{i} \in \partial T: \Delta_{i}>k C\right\}\right) \leq \int_{\partial T}\left(\Delta_{i}+C\right) v(\mathrm{~d} \boldsymbol{i})=\int_{\partial T} \Delta_{i} v(\mathrm{~d} \boldsymbol{i})+C Y .
$$


Proof of Lemma 8.7. We have

$$
\nu\left(\left\{i \in \partial T: \limsup _{n \rightarrow \infty} \frac{\Delta_{i}^{i \mid k}}{k}>\epsilon\right\}\right) \leq \lim _{l \uparrow \infty} \sum_{k \geq l} v\left(\left\{i \in \partial T: \Delta_{i}^{i \mid k}>k \epsilon\right\}\right) .
$$

Now

$$
\left\{\boldsymbol{i} \in \partial T: \Delta_{i}^{i \mid k}>k \epsilon\right\}=\left\{\boldsymbol{j} \in T_{k}, \boldsymbol{j} \boldsymbol{i} \in \partial T: \Delta_{j i}^{j}>k \epsilon\right\},
$$

and, for $j \in T_{k}$,

$$
\begin{aligned}
\mathrm{E}\left[v\left(\left\{\boldsymbol{j} \boldsymbol{i} \in \partial T: \Delta_{j \boldsymbol{i}}^{j}>k \epsilon\right\}\right) \mid \mathscr{B}_{k}\right] & =y_{\boldsymbol{j}} \mathrm{E}\left[v_{\boldsymbol{j}}\left(\left\{\boldsymbol{j} \boldsymbol{i} \in \partial T: \Delta_{\boldsymbol{j} \boldsymbol{i}}^{\boldsymbol{j}}>k \epsilon\right\}\right)\right] \\
& =y_{\boldsymbol{j}} \mathrm{E}\left[v\left(\left\{\boldsymbol{i} \in \partial T: \Delta_{\boldsymbol{i}}>k \epsilon\right\}\right)\right] .
\end{aligned}
$$

Hence,

$$
\mathrm{E}\left[v\left(\left\{\boldsymbol{i} \in \partial T: \Delta_{i}^{i \mid k}>k \epsilon\right\}\right) \mid \mathscr{B}_{k}\right]=\sum_{\boldsymbol{j} \in T_{k}} y_{j} \operatorname{Ev}\left(\left\{\boldsymbol{i} \in \partial T: \Delta_{i}>k \epsilon\right\}\right),
$$

and so

$$
\mathrm{E}\left[v\left(\left\{\boldsymbol{i} \in \partial T: \Delta_{i}^{i \mid k}>k \epsilon\right\}\right)\right]=\operatorname{Ev}\left(\left\{\boldsymbol{i} \in \partial T: \Delta_{i}>k \epsilon\right\}\right) .
$$

By Lemmas 8.8 and 8.9, these have a finite sum over $k$. Hence, for any $\epsilon>0$,

$$
\operatorname{Ev}\left(\left\{i \in \partial T: \limsup _{n \rightarrow \infty} \frac{\Delta_{i}^{i \mid k}}{k}>\epsilon\right\}\right)=0
$$

and so $\operatorname{Ev}\left(\partial T \backslash G_{5}\right)=0$.

Proof of Proposition 8.1. Combine the sets introduced in Lemmas 8.3, 8.4, 8.6, and 8.7 by letting $G^{*}=G_{1} \cap G_{2} \cap G_{3} \cap G_{4} \cap G_{5}$. For $i \in G^{*}$ and $\varrho<\mathscr{D}\left(\pi(\boldsymbol{i}), V^{\mathrm{c}}\right)$, let $k=k(\varrho)$ be the largest integer such that

$$
\varrho<\mathscr{D}\left(\pi(\boldsymbol{i}), V_{i \mid k}^{\mathrm{c}}\right)\left(=\exp \left(-\Delta_{i}^{i \mid k}-\sigma_{i \mid k}\right)\right) .
$$

Then

$$
B(\pi(\boldsymbol{i}) ; \varrho) \subset V_{i \mid k},
$$

and so, using Lemma 7.2 or 7.3 as appropriate,

$$
\nu_{\pi}(B(\pi(\boldsymbol{i}) ; \varrho)) \leq y_{i \mid k} Y_{i \mid k} .
$$

Now, by arrangement (with $k=k(\varrho)$ ) we have

$$
\Delta_{i}^{i \mid k}+\sigma_{i \mid k}<-\log \varrho \leq \Delta_{i}^{i \mid(k+1)}+\sigma_{i \mid(k+1)} .
$$

Since $i \in G^{*} \subset G_{3} \cap G_{5}$, Lemmas 8.6 and 8.7 give, as $\varrho \rightarrow 0$,

$$
\frac{-\log \varrho}{k} \rightarrow \widetilde{\mathrm{E}} S_{1}=-\mathrm{E}\left[\sum_{i} p_{i}^{q} r_{i}^{\beta} \log r_{i}\right]
$$

and so, using also Lemma 8.3 and $i \in G^{*} \subset G_{1} \cap G_{3} \cap G_{4} \cap G_{5}$,

$$
\frac{q \log p_{i \mid k}+\beta \log r_{i \mid k}+\log Y_{i \mid k}}{\log \varrho} \rightarrow q \alpha+\beta \quad \text { as } \varrho \rightarrow 0 .
$$


Now substituting for $y_{i \mid k}$ into (8.2) gives

$$
\frac{\nu_{\pi}(B(\pi(\boldsymbol{i}) ; \varrho))}{\varrho^{s}} \leq \exp \left(\left(\frac{q \log p_{\boldsymbol{i} \mid k}+\beta \log r_{\boldsymbol{i} \mid k}+\log Y_{\boldsymbol{i} \mid k}}{\log \varrho}-s\right) \log \varrho\right),
$$

which is bounded as $\varrho \downarrow 0$ for $s<q \alpha+\beta$. Hence, Lemma 8.2 gives $\operatorname{dim}_{H}\left(\pi\left(G^{*}\right)\right) \geq q \alpha+\beta$ and Lemma 8.1 notes that $\alpha q+\beta=\beta^{*}(\alpha)$.

It remains to show that $\pi\left(G^{*}\right) \subset F_{\alpha}$. Let $r=r(\varrho)$ be the smallest integer such that $\varrho>\exp \left(-\sigma_{i \mid r}\right)$. Then, with $k$ as already defined,

$$
K_{i \mid r} \subset B(\pi(\boldsymbol{i}) ; \varrho) \subset V_{\boldsymbol{i} \mid k},
$$

so

$$
\mu\left(C_{\boldsymbol{i} \mid r}\right) \leq \mu_{\pi}\left(K_{\boldsymbol{i} \mid r}\right) \leq \mu_{\pi}(B(\pi(\boldsymbol{i}) ; \varrho)) \leq \mu_{\pi}\left(V_{\boldsymbol{i} \mid k}\right),
$$

which combines with Lemma 2.6 or 7.2 to give

$$
\log p_{i \mid r}+\log W_{i \mid r} \leq \log \mu_{\pi}(B(\pi(\boldsymbol{i}) ; \varrho)) \leq \log p_{\boldsymbol{i} \mid k}+\log W_{i \mid k}
$$

Now, by arrangement we have

$$
\sigma_{i \mid(r-1)} \leq-\log \varrho<\sigma_{i \mid r}
$$

and so, since $i \in G^{*} \subset G_{3}$, as $\varrho \rightarrow 0$,

$$
\frac{-\log \varrho}{r} \rightarrow \widetilde{\mathrm{E}} S_{1}
$$

Dividing (8.4) through by $\log \varrho$ and letting $\varrho \downarrow 0$, using (8.3) and (8.5) and Lemmas 8.4 and 8.6,

$$
\frac{\log \mu_{\pi}(B(\pi(\boldsymbol{i}) ; \varrho))}{\log \varrho} \rightarrow \alpha
$$

for $i \in G_{2} \cap G_{3} \cap G_{4} \cap G_{5}$. Hence, $\pi\left(G^{*}\right) \subset F_{\alpha}$, as required.

\section{Moments of $W$}

The results impose moment conditions on $W$ and $Y$ (which is also a ' $W$ '). For applications, these need to be traced back to conditions on the scaling law. Since $\mathrm{E} W=1$, it is automatic that $\mathrm{E} W^{q}<\infty$ for $0 \leq q \leq 1$. For the other cases, various suitable results are known. Typical results can be found in [3], [16], [23], and [24]. It is worth saying that, by formulating using point process language, several early relevant papers allowed $\mathrm{P}(N=\infty)>0$. We record several such results. Recall that (2.3) is in force throughout to ensure that $\mathrm{E} W=1$, and Assumption B(i) and (ii) play the same role for $Y$.

Lemma 9.1. (Proposition 4 of [16].) For $q>1, \mathrm{E} W^{q}$ is finite when

$$
\mathrm{E}\left[\left(\sum p_{i}\right)^{q}\right]<\infty \text { and } m(q, 0)=\mathrm{E}\left[\sum p_{i}^{q}\right]<1 .
$$

Lemma 9.2. (Theorem 2.4 of [24].) Assume that $\mathrm{P}(1 \leq N<\infty)=1$. For $q<0$, EW $W^{q}$ is finite when, for some $\epsilon>0$,

$$
\mathrm{E}\left[\left(\max p_{i}\right)^{q-\epsilon}\right]<\infty \text { and } \mathrm{E}\left[p_{1}^{q-\epsilon} I(N=1)\right]<1 .
$$


Lemma 9.3. (Page 28 of [6].) It holds that $\mathrm{E} W \log W$ is finite when

$$
\mathrm{E}\left[\left(\sum p_{i}\right)\left(\log \left(\sum p_{i}\right)\right)^{2}\right]<\infty .
$$

Simplifications of Lemmas 9.1 and 9.2 are worth recording.

Lemma 9.4. For $q>1, \mathrm{EW}^{q}$ is finite when $p_{i} \in(0,1)$ for all $i$ and

$$
\mathrm{E}\left[\left(\sum p_{i}\right)^{q}\right]<\infty .
$$

Lemma 9.5. For $q<0, \mathrm{E}^{q}$ is finite when, for some $\epsilon>0$,

$$
m(q-\epsilon, 0)=\mathrm{E}\left[\sum p_{i}^{q-\epsilon}\right]<\infty \text { and } \mathrm{E}\left[p_{1}^{q-\epsilon} I(N=1)\right]<1
$$

Lemmas 9.1-9.5 cover the moment conditions needed on branching process limits $(Y$ and $W$ ) in Theorem 3.1 except for (3.8). The following lemma brings this condition also into the range of those lemmas.

Lemma 9.6. It holds that $\mathrm{E} Y|\log W|$ is finite whenever there is a $\delta>0$ for which $\mathrm{E} Y^{1+\delta}$ and $\mathrm{E} W^{-\delta}$ are both finite.

Proof. Take $\delta<1$. For any $\epsilon>0$, there is a $C$ such that

$$
Y|\log W| \leq C Y\left(W^{\epsilon}+W^{-\epsilon}\right),
$$

and Hölder's inequality gives

$$
\mathrm{E} Y W^{-\epsilon} \leq\left(\mathrm{E} Y^{1+\delta}\right)^{1 /(1+\delta)}\left(\mathrm{E} W^{-\epsilon(1+\delta) / \delta}\right)^{\delta /(1+\delta)} \leq\left(\mathrm{E} Y^{1+\delta}\right)^{1 /(1+\delta)}\left(\mathrm{E} W^{-\delta}\right)^{\delta /(1+\delta)}
$$

for small enough $\epsilon$. Since $\mathrm{E} W^{\delta}$ is always finite for $\delta \leq 1, \mathrm{E} Y W^{\epsilon}$ is bounded in the same way.

\section{Simplifying conditions}

Theorem 3.1 has a complex collection of conditions. Here we indicate some simpler sufficient ones.

Theorem 10.1. Suppose that the open set condition and Assumption A both hold and that $K$ has a $\gamma$-boundary. Suppose also that $m(q, \beta)$ is finite for all $(q, \beta)$. Let $\alpha \in\left(D_{L}, D_{U}\right)$, with $\tilde{q}(\alpha)$ and $\beta(\tilde{q}(\alpha))$ as in Lemma 3.2(vi). Assume that (3.4) holds and, if $\tilde{q}(\alpha)>1$, assume that (3.6) holds. Then

$$
\operatorname{dim}_{H}\left(F_{\alpha}\right) \leq \beta^{*}(\alpha) \text { a.s. }
$$

Furthermore, if $\beta^{*}(\alpha)<0$ then $F_{\alpha}=\varnothing$ a.s. When $\beta^{*}(\alpha)>0$, Assumption $B$ holds for $(\tilde{q}(\alpha), \beta(\tilde{q}(\alpha)))$. Suppose also that (3.7) and (3.8) hold. Then

$$
\operatorname{dim}_{H}\left(F_{\alpha}\right)=\beta^{*}(\alpha) \text { a.s. }
$$

Proof. When $m(q, \beta)$ is finite for all $(q, \beta),(3.5)$ always holds, as do Assumption B(iii) and (iv), and $N$ is always finite. Furthermore, $m(q, \beta(q))=1, \alpha=P(q, \beta(q)) / R(q, \beta(q))$ is finite, in $\left(D_{L}, D_{U}\right)$, and satisfies $\beta^{*}(\alpha)=q \alpha+\beta(q)$, and every $\alpha$ in $\left(D_{L}, D_{U}\right)$ can be obtained in this way. Thus, Assumption B(ii) also holds when $(\tilde{q}(\alpha), \beta(\tilde{q}(\alpha)))$ give $\beta^{*}(\alpha)>0$. 
This still leaves the rather complicated moment conditions on $W$ and $Y$ and (3.6). To give a simpler version that covers these, recall that $N$ is the family size and let

$$
\sigma_{*}=\max _{i}\left\{-\log r_{i}\right\}, \quad z_{*}=\max _{i}\left\{-\log p_{i}\right\}, \quad \text { and } \quad z^{*}=\max _{i}\left\{\log p_{i}\right\},
$$

so that $\left(-z^{*}\right) \leq z_{*}$.

Lemma 10.1. For $\varrho \geq 1$, suppose that, for some $\epsilon>0$, E $N^{\varrho+\epsilon}<\infty$, and that $\sigma_{*}, z_{*}$, and $z^{*}$ all have moment generating functions that are finite for all positive arguments. Then

$$
\mathrm{E}\left[\left(\sum_{i} p_{i}^{q} r_{i}^{\beta}\right)^{\varrho}\right]<\infty
$$

In particular, $\varrho=1$ gives that $m(q, \beta)$ is finite for all $(q, \beta)$.

Proof. Note that

$$
\mathrm{E}\left[\left(\sum_{i} p_{i}^{q} r_{i}^{\beta}\right)^{\varrho}\right] \leq \mathrm{E}\left[\left(N \mathrm{e}^{-\sigma_{*} \beta I(\beta<0)} \mathrm{e}^{-z_{*} q I(q<0)} \mathrm{e}^{z^{*} q I(q>0)}\right)^{\varrho}\right],
$$

and then several applications of Hölder's inequality gives the result.

Lemma 10.2. For any $q>1,(3.6)$ holds with $\tilde{q}(\alpha)=q$ and any positive $\gamma$ if $\sigma_{*}$ has a moment generating functions that is finite for all positive arguments and, for some $\epsilon>0, \mathrm{E} W^{q+\epsilon}<\infty$.

Proof. Hölder's inequality shows that (3.6) holds with $\tilde{q}(\alpha)=q$ provided that $\mathrm{E}\left[\left(\sum p_{i}\right)^{q+\epsilon}\right]$ is finite for some $\epsilon>0$. By martingale properties,

$$
\mathrm{E}\left[\left(\sum_{i} p_{i}\right)^{q+\epsilon}\right] \leq \mathrm{E}\left[\left(\sum_{i \in T_{n}} p_{i}\right)^{q+\epsilon}\right] \leq \mathrm{E} W^{q+\epsilon} .
$$

Lemma 10.3. Suppose that $\mathrm{E} N^{1+\epsilon}<\infty$ and that $\left(-z^{*}\right)$ has a moment generating function that is finite for all positive arguments. Suppose too that $\mathrm{P}(N \geq 2)=1$. Then $\mathrm{E} W^{q}<\infty$ for $q<0$.

Proof. Since $\mathrm{P}(N \geq 2)=1, \mathrm{E}\left[p_{1}^{q-\epsilon} I(N=1)\right]=0$. Now Lemma 9.5 gives the result.

Lemma 10.4. Suppose that $(q, \beta)$ are such that $m(q, \beta)=\mathrm{E} \sum_{i} p_{i}^{q} r_{i}^{\beta}=1, m$ is finite in a neighbourhood of $(q, \beta)$,

$$
-\mathrm{E} \sum_{i} p_{i}^{q} r_{i}^{\beta}\left(q \log p_{i}+\beta \log r_{i}\right)>0
$$

and

$$
\mathrm{E}\left[\left(\sum_{i} p_{i}^{q} r_{i}^{\beta}\right)^{1+\epsilon}\right]<\infty
$$

Then $\mathrm{E} Y^{(1+\epsilon)}<\infty$.

Proof. Note that

$$
\mathrm{E}\left[\sum_{i}\left(p_{i}^{q} r_{i}^{\beta}\right)^{1+\epsilon}\right]=m(q(1+\epsilon), \beta(1+\epsilon))<1
$$

for sufficiently small $\epsilon$ by calculus, using (10.1). Lemma 9.1 gives the result. 
Lemma 10.5. For $\epsilon>0$, suppose that $\mathrm{E} N^{1+2 \epsilon}<\infty$, and that $\sigma_{*}, z_{*}$, and $z^{*}$ all have moment generating functions that are finite for all positive arguments. Suppose that $(q, \beta)$ are such that $m(q, \beta)=\mathrm{E} \sum_{i} p_{i}^{q} r_{i}^{\beta}=1$ and (10.1) holds. Then $\mathrm{E} Y^{(1+\epsilon)}<\infty$.

Proof. Lemma 10.1 applies to show that $m$ is finite everywhere and that (10.2) holds. Now Lemma 10.4 gives the result.

Proof of Corollary 3.1. This is an application of Theorem 3.1. It is easy to check that Assumption A holds and, as noted after Definition 3.1, $K$ has a $d$-boundary. By assumption, each of

$$
\mathrm{Ee}^{\varrho \sigma_{*}}=\mathrm{E}\left(\min _{i} r_{i}\right)^{-\varrho}, \quad \mathrm{Ee}^{\varrho z_{*}}=\mathrm{E}\left(\min _{i} p_{i}\right)^{-\varrho}, \quad \mathrm{Ee}^{\varrho z^{*}}=\mathrm{E}\left(\max _{i} p_{i}\right)^{\varrho}
$$

is finite for all $\varrho>0$ and $\mathrm{E} N^{1+\epsilon}<\infty$. Hence, by Lemma 10.1, $m$ is finite everywhere. Thus, (3.5) and parts (iii) and (iv) of Assumption B hold when needed.

Turning to conditions on $W$, by Lemma 9.4, E, $W^{q}$ is finite for all $q>1$, and this implies also that $\mathrm{E} W^{q}$ is finite for $0 \leq q \leq 1$. By Lemma 10.3, $\mathrm{E} W^{q}$ is finite for all $q<0$. Hence, (3.4) holds. Lemma 10.2 shows that (3.6) also holds. For the conditions on $Y$, Lemma 10.5 shows that, for small positive $\eta, \mathrm{E} Y^{1+\eta}<\infty$, which implies that Assumption B(ii) and (3.7) hold. Furthermore, Lemma 9.6 implies that (3.8) holds.

\section{An example}

In [14, Section 6.2] the description of an optimal path in a very heavy tailed last passage percolation problem is formulated in terms of a random self-similar measure. This measure was not covered by the results then available in the literature. The example is easy to cast into the current notation. Let $V$ and $\tilde{V}$ be independent uniform random variables. Now let $N=2,\left(p_{1}, p_{2}\right)=(\tilde{V}, 1-\tilde{V}), K=[0,1]$, and $\left(K_{1}, K_{2}\right)=([0, V],[V, 1])$ so that $\left(r_{1}, r_{2}\right)=(V, 1-V)$. As computed in [14],

$$
m(q, \beta)=\frac{2}{(1+q)(1+\beta)} \text { for } q>-1, \beta>-1,
$$

so $\beta(q)=(1-q) /(1+q)$ and

$$
\beta^{*}(\alpha)=\alpha \tilde{q}(\alpha)+\beta(\tilde{q}(\alpha)) \quad \text { with } \quad \tilde{q}(\alpha)=-1+\sqrt{\frac{2}{\alpha}} .
$$

The example satisfies the open set condition and $K$ has 0-boundary, since its boundary is always covered by at most two disjoint balls. It is easy to see that Assumption A holds.

Since $\sum p_{i}=1$, Lemma 9.1 implies that $\mathrm{E} W^{q}<\infty$ for all $q>1$. Also, max $p_{i}$ is uniform on $[0.5,1]$ and so has finite moments of all orders and $N=2$. Hence, Lemma 9.2 shows that E $W^{q}<\infty$ for all $q<0$. Therefore, (3.4) holds.

Since $\min r_{i}$ is uniform on $[0,0.5], \mathrm{E}\left(\min r_{i}\right)^{\delta}$ is finite for $\delta>-1$, and, as already noted, $\sum p_{i}=1$. Furthermore, when $\tilde{q}(\alpha) \in(1, \infty), \beta(\tilde{q}(\alpha)) \in(-1,0)$. Hence, (3.6) holds when $\tilde{q}(\alpha)>1$.

Fix $\alpha \in(0, \infty)$ with $\beta^{*}(\alpha)=\alpha \tilde{q}(\alpha)+\beta(\tilde{q}(\alpha))>0$. A verification that, for some $\epsilon>0$, $\mathrm{E} Y^{1+\epsilon}<\infty$ is in [14] (where $Y$ is $W(\tilde{q}(\alpha))$ there). Alternatively, $m$ is finite in a neighbourhood of $\left(\tilde{q}(\alpha), \beta(\tilde{q}(\alpha))\right.$, and reversing the argument for Lemma 8.1, $\beta^{*}(\alpha)>0$ implies that (10.1) 
holds for $(\tilde{q}(\alpha), \beta(\tilde{q}(\alpha))$. Furthermore, for any $q>-1$ and $\beta>-1$, and sufficiently small $\epsilon$,

$$
\begin{aligned}
\mathrm{E}\left[\left(\sum p_{i}^{q} r_{i}^{\beta}\right)^{1+\epsilon}\right] & \leq \mathrm{E}\left[\left(2\left(\max p_{i}^{q}\right)\left(\max r_{i}^{\beta}\right)\right)^{1+\epsilon}\right] \\
& \leq 2^{1+\epsilon} \mathrm{E}\left[\max p_{i}^{q(1+\epsilon)}\right] \mathrm{E}\left[\max r_{i}^{\beta(1+\epsilon)}\right] \\
& <\infty .
\end{aligned}
$$

Hence, (10.2) also holds and then Lemma 10.4 gives $\mathrm{E} Y^{1+\epsilon}<\infty$ and, hence, (3.7). Earlier in this proof it was established that, for any $\epsilon>0, \mathrm{E} W^{-\epsilon}<\infty$ and so now Lemma 9.6 shows that (3.8) holds. Thus, all the conditions in Theorem 3.1 hold. The multifractal spectrum for this example was computed in [14], based on a preliminary version of this study. Unfortunately, Lemma 6.6 there, which is supposed to be here, has turned out to have some conditions missing, as can be seen by comparing it with Theorem 3.1. Happily, the omissions do not invalidate the assertions made about the example.

\section{References}

[1] Alsmeyer, G. And Iksanov, A. M. (2009). A log-type moment result for perpetuities and its application to martingales in supercritical branching random walks. Electron J. Prob. 14, 289-312.

[2] Arbeiter, M. and PatzschKe, N. (1996). Random self-similar multifractals. Math. Nachr. 181, 5-42.

[3] Barral, J. (2000). Continuity of the multifractal spectrum of a random statistically self-similar measure. J. Theoret. Prob. 13, 1027-1060.

[4] Barral, J. (2001). Generalized vector multiplicative cascades. Adv. Appl. Prob. 33, 874-895.

[5] Biggins, J. D. (1977). Martingale convergence in the branching random walk. J. Appl. Prob. 14, 25-37.

[6] Biggins, J. D. (1979). Growth rates in the branching random walk. Z. Wahrscheinlichkeitsth. 48, 17-34.

[7] Biggins, J. D. (1995). The growth and spread of the general branching random walk. Ann. Appl. Prob. 5, 1008-1024.

[8] Biggins, J. D. (1998). Lindley-type equations in the branching random walk. Stoch. Process. Appl. 75, 105-133.

[9] Biggins, J. D. And Kyprianou, A. E. (1997). Seneta-Heyde norming in the branching random walk. Ann. Prob. 25, 337-360.

[10] Biggins, J. D. and Kyprianou, A. E. (2004). Measure change in multitype branching. Adv. Appl. Prob. 36, 544-581.

[11] Chow, Y. S. And Teicher, H. (1978). Probability Theory. Springer, New York.

[12] Falconer, K. (1990). Fractal Geometry. John Wiley, Chichester.

[13] Falconer, K. J. (1994). The multifractal spectrum of statistically self-similar measures. J. Theoret. Prob. 7, 681-702.

[14] Hambly, B. And Martin, J. B. (2007). Heavy tails in last-passage percolation. Prob. Theory Relat. Fields 137, 227-275.

[15] Hutchinson, J. E. And Rüschendorf, L. (2000). Random fractals and probability metrics. Adv. Appl. Prob. 32, 925-947.

[16] IKsanov, A. M. (2004). Elementary fixed points of the BRW smoothing transforms with infinite number of summands. Stoch. Process. Appl. 114, 27-50.

[17] Jagers, P. (1975). Branching Processes with Biological Applications. Wiley-Interscience, London.

[18] Jagers, P. (1989). General branching processes as Markov fields. Stoch. Process. Appl. 32, 183-212.

[19] Kolumbán, J., Soós, A. And Varga, I. (2003). Self-similar random fractal measures using contraction method in probabilistic metric spaces. Internat. J. Math. Math. Sci. 2003, 3299-3313.

[20] LataŁA, R. (1997). Estimation of moments of sums of independent real random variables. Ann. Prob. 25, 1502-1513.

[21] Liang, J.-R. (2002). Random Markov-self-similar measures. Stoch. Process. Appl. 98, 113-130.

[22] LIU, Q. (1998). Fixed points of a generalized smoothing transformation and applications to the branching random walk. Adv. Appl. Prob. 30, 85-112.

[23] LIU, Q. (2000). On generalized multiplicative cascades. Stoch. Process. Appl. 86, 263-286.

[24] LIU, Q. (2001). Asymptotic properties and absolute continuity of laws stable by random weighted mean. Stoch. Process. Appl. 95, 83-107. 
[25] Liu, Q. AND Rouault, A. (1997). On two measures defined on the boundary of a branching tree. In Classical and Modern Branching Processes (Minneapolis, MN, 1994; IMA Vol. Math. Appl. 84), Springer, New York, pp. 187-201.

[26] LyONs, R. (1997). A simple path to Biggins' martingale convergence for branching random walk. In Classical and Modern Branching Processes (Minneapolis, MN, 1994; IMA Vol. Math. Appl. 84), Springer, New York, pp. 217-221.

[27] Mauldin, R. D. and Williams, S. C. (1986). Random recursive constructions: asymptotic geometric and topological properties. Trans. Amer. Math. Soc. 295, 325-346.

[28] Nerman, O. (1981). On the convergence of supercritical general (C-M-J) branching processes. Z. Wahrscheinlichkeitsth. 57, 365-395.

[29] OlsEN, L. (1994). Random Geometrically Graph Directed Self-Similar Multifractals (Pitman Res. Notes Math. Ser. 307), Longman Scientific \& Technical, Harlow.

[30] Olsen, L. (1995). A multifractal formalism. Adv. Math. 116, 82-196.

[31] Patzschke, N. (1997). The strong open set condition in the random case. Proc. Amer. Math. Soc. 125, 21192125.

[32] PatzschKe, N. And Zähle, U. (1990). Self-similar random measures. IV. The recursive construction model of Falconer, Graf, and Mauldin and Williams. Math. Nachr. 149, 285-302.

[33] Rockafellar, R. T. (1970). Convex Analysis (Princeton Math. Ser. 28). Princeton University Press.

[34] ZäHLE, U. (1988). Self-similar random measures. I. Notion, carrying Hausdorff dimension, and hyperbolic distribution. Prob. Theory Relat. Fields 80, 79-100. 